\title{
Investigation of the $\mathrm{v} 2+2 \mathrm{v} 3$
}

Subband in the Overtone Icosad

of $13 \mathrm{CH} 4$ by Pulsed Supersonic

Jet and Diode Laser Cavity Ring-

Down Spectroscopy: Partial

Rovibrational Analysis and Nuclear

Spin Symmetry Conservation

\section{Journal Article}

Author(s):

Bjelobrk, Zoran; Manca Tanner, Carine; Quack, Martin

Publication date:

2015-10

Permanent link:

https://doi.org/10.3929/ethz-b-000104993

Rights / license:

In Copyright - Non-Commercial Use Permitted

Originally published in:

Zeitschrift für physikalische Chemie 229(10-12), https://doi.org/10.1515/zpch-2015-0635 
Funding acknowledgement:

290925 - Spectroscopy, Quantum Dynamics and Electroweak Parity Violation in Chiral Molecules (EC)

This page was generated automatically upon download from the ETH Zurich Research Collection. For more information, please consult the Terms of use. 
Zoran Bjelobrk, Carine Manca Tanner*, and Martin Quack* Investigation of the $v_{2}+2 v_{3}$ Subband in the Overtone Icosad of ${ }^{13} \mathrm{CH}_{4}$ by Pulsed Supersonic Jet and Diode Laser Cavity Ring-Down Spectroscopy: Partial Rovibrational Analysis and Nuclear Spin Symmetry Conservation

DOI 10.1515/zpch-2015-0635

Received April 28, 2015; accepted July 28, 2015

Abstract: We have investigated the $v_{2}+2 v_{3}$ combination band of methane ${ }^{13} \mathrm{CH}_{4}$ centered at $7493.15918 \mathrm{~cm}^{-1}$ within the icosad of the overtone absorption. The jet-CRD setup combining supersonic jet expansions and cavity ring-down spectroscopy which was already used for the reinvestigation of the same spectral region for the main isotope of methane $\left({ }^{12} \mathrm{CH}_{4}\right)$ has been used to record spectra of the $Q$ and $R$ branches at room temperature as well as at very low temperatures (down to $4 \mathrm{~K}$ ). Based on our previous temperature-dependent investigations and the present results, we provide a careful analysis and the assignment for lines involving angular momentum quantum numbers up to $J=4$. The analysis of the relative intensities in spectra taken at various rotational and effective translational temperatures demonstrate conservation of nuclear spin symmetry for ${ }^{13} \mathrm{CH}_{4}$ under the conditions of a supersonic jet expansion, similar to our previous results regarding ${ }^{12} \mathrm{CH}_{4}$ and also to further results using other techniques and covering other spectral ranges. This is in agreement with theoretical expectation regarding very slow nuclear spin symmetry relaxation under these conditions in supersonic jet expansions.

Keywords: High Resolution Spectroscopy, Near Infrared, Cavity Ring Down Spectroscopy, Methane, Isotopes, ${ }^{13} \mathrm{CH}_{4}$, Nuclear Spin Symmetry.

*Corresponding authors: Carine Manca Tanner, Laboratorium für Physikalische Chemie, ETH Zürich, CH-8093 Zürich, Switzerland, e-mail: carine.manca@ir.phys.chem.ethz.ch and Martin Quack, Laboratorium für Physikalische Chemie, ETH Zürich, CH-8093 Zürich, Switzerland, e-mail: quack@ir.phys.chem.ethz.ch

Zoran Bjelobrk: Laboratorium für Physikalische Chemie, ETH Zürich, CH-8093 Zürich, Switzerland 
Dedicated to Prof. Dr. Dr. h.c. mult. Jürgen Troe on the occasion of his $75^{\text {th }}$ birthday

\section{Introduction}

Methane is of great interest as a prototype for structure and dynamics of simple organic molecules [1, 2] and fundamental questions ranging from formulations of global multidimensional potential energy surfaces including stereomutation and dissociation [3-17] to accurate variational energy level calculations on such surfaces [18-21], intramolecular vibrational energy flow [7, 8, 22-25] to finally tiny effects such as parity violation [26-28]. It is furthermore of the greatest importance in applied areas such as combustion of natural gas, atmospheric chemistry and spectroscopy, and the Earth's climate where it contributes importantly to the Greenhouse-Effect [29]. It is also of interest in the spectroscopy of planetary atmospheres [30, 31]. While the main isotopomer ${ }^{12} \mathrm{CH}_{4}$ has been subject to substantial spectroscopic efforts including recent analyses up to the octad [32, 33] (up to $4700 \mathrm{~cm}^{-1}$ ) and a partial analysis up to the tetradecad ranges [34] (up to $6250 \mathrm{~cm}^{-1}$ ), much less is known about the spectra of the second most important isotopomer ${ }^{13} \mathrm{CH}_{4}$ with about $1 \%$ natural abundance (see [35-38]).

Table 1 provides a survey of the fundamentals of ${ }^{12} \mathrm{CH}_{4}$ and ${ }^{13} \mathrm{CH}_{4}$. As is well known [32, 36, 39] because of the approximate relation:

$$
v_{1}\left(\mathrm{~A}_{1}\right) \simeq v_{3}\left(\mathrm{~F}_{2}\right) \simeq 2 v_{2}(\mathrm{E}) \simeq 2 v_{4}\left(\mathrm{~F}_{2}\right)
$$

the infrared spectrum and "quasidegenerate" energy levels of methane can be grouped into so-called polyads $\mathrm{P}_{n}$ with an integer polyad quantum number:

Table 1: Band center $\tilde{v}_{0}$ (in cm ${ }^{-1}$ ) of the fundamentals $v_{i}$ of ${ }^{12} \mathrm{CH}_{4}$ and of ${ }^{13} \mathrm{CH}_{4}$ and their symmetry species in the $T_{\mathrm{d}}$ point group and the approximate description of the normal mode motions. The values are current best values (rounded to two digits) from References [32, 36, 38] but we also give some previous relevant references providing data of lower accuracy (see also [47]).

\begin{tabular}{lrrrr}
\hline Vibration & $\Gamma\left(T_{\mathrm{d}}\right)$ & Assignment & $\tilde{v}_{0} / \mathrm{cm}^{-1}$ & ${ }^{12} \mathrm{CH}_{4}$ \\
\hline$v_{1}$ & $\mathrm{~A}_{1}$ & symmetric CH-stretching & $2916.48[32,40-42]$ & $2915.44[38]$ \\
$v_{2}$ & $\mathrm{E}$ & $\mathrm{C}$-bending & $1533.33[32,40,42,43]$ & $1533.49[38]$ \\
$v_{3}$ & $\mathrm{~F}_{2}$ & $\mathrm{CH}$-stretching & $3019.49[32,40,42,44]$ & $3009.55[35,38]$ \\
$v_{4}$ & $\mathrm{~F}_{2}$ & $\mathrm{CH}$-bending & $1310.76[32,40,42,43]$ & $1302.78[35,38]$ \\
\hline
\end{tabular}


Table 2: Polyads of methane (up to the Icosad) labelled with the polyad labels $\mathrm{P}_{n}$, as well as the approximate spectral ranges for ${ }^{13} \mathrm{CH}_{4}$.

\begin{tabular}{llrrr}
\hline$P_{n}$ & $\begin{array}{l}\text { Name of } \\
\text { the polyad }\end{array}$ & $\begin{array}{r}\text { Number of } \\
\text { levels }\end{array}$ & $\begin{array}{r}\text { Number of } \\
\text { sublevels }\end{array}$ & $\begin{array}{r}\text { Spectral range } \\
\left(\text { in cm }^{-1}\right)\end{array}$ \\
\hline$P_{0}$ & Monad & 1 & 1 & 0 \\
$P_{1}$ & Dyad & 2 & 2 & $1200-1630$ \\
$P_{2}$ & Pentad & 5 & 9 & $2470-3170$ \\
$P_{3}$ & Octad & 8 & 24 & $3750-4700$ \\
$P_{4}$ & Tetradecad & 14 & 60 & $5000-6230$ \\
$P_{5}$ & Icosad & 20 & 134 & $6230-7700$ \\
\hline
\end{tabular}

$$
n=2 v_{1}+v_{2}+2 v_{3}+v_{4}
$$

where $v_{i}$ represents the quantum number for the normal vibration $v_{i}$. The names of the polyads are derived from the Greek prefix for the number of levels in each polyad, which are further split into sublevels of well defined symmetry species in the point group $T_{\mathrm{d}}$ (see Section 3.1). Table 2 gives a summary of the levels and sublevels up to the Icosad, which is the current frontier for analyses that are very fragmentary, so far for the icosad and higher polyads [36].

In previous work we have already studied the subband $v_{2}+2 v_{3}$ of the icosad of ${ }^{12} \mathrm{CH}_{4}$ in some detail [45-47] and we refer to these papers for further background discussion and references. Supersonic jet-cavity ring-down spectroscopy has proven to be avery powerful tool to provide a first analysis. One interesting further question, which can be studied by supersonic jet spectroscopy both of the infrared spectra in the fundamental and overtone ranges concerns the question of nuclear spin symmetry conservation: do the relative population of the nuclear spin symmetry isomers A (meta), E (para) and F (ortho) for methane (analogous to ortho and para nuclear spin isomers of hydrogen $\mathrm{H}_{2}$ ) retain their high (room) temperature populations upon cooling due to nuclear spin symmetry conservation $[57,58]$ or do they relax to their true equilibrium values at low temperatures (nuclear spin symmetry relaxation)? Experiments on supersonic jet expansions of ${ }^{12} \mathrm{CH}_{4}$ have invariably shown nuclear spin symmetry conservation [48-50], whereas under room temperature conditions at high pressures and in matrices one observes nuclear spin symmetry relaxation on time scales of the order of seconds to hours [51, 52]. No such studies exist yet for ${ }^{13} \mathrm{CH}_{4}$ to our knowledge. They might, however, be of some interest because of the possible influence of the ${ }^{13} \mathrm{C}$ nuclear spin $1 / 2$ and negative parity, different from the ${ }^{12} \mathrm{C}$ nucleus without spin and positive parity, and because of the changes in energy level structure between ${ }^{12} \mathrm{CH}_{4}$ and ${ }^{13} \mathrm{CH}_{4}$ which 

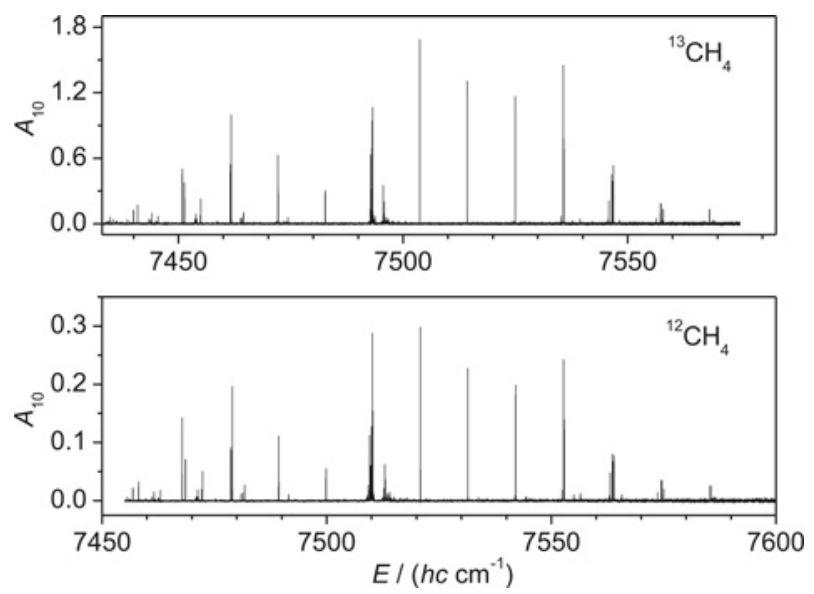

Figure 1: FTIR survey spectrum of the $v_{2}+2 v_{3}$ combination band of (top) ${ }^{13} \mathrm{CH}_{4}$ and (bottom) ${ }^{12} \mathrm{CH}_{4}$ recorded at $80 \mathrm{~K}$ and shown in decadic absorbance $A_{10}=\log _{10}\left(I_{0} / I\right)$. The conditions correspond to an optical path length $\ell=10 \mathrm{~m}$ and partial pressure $p\left({ }^{13} \mathrm{CH}_{4}\right)=28 \mathrm{~Pa}$ in a $10 \%$ mixture with $\mathrm{He}$, and $\ell=5 \mathrm{~m}$ and $p\left({ }^{12} \mathrm{CH}_{4}\right)=49 \mathrm{~Pa}$ (see Reference [36] and also [47]). The wavenumber scale is shifted by $\Delta \tilde{v}=17 \mathrm{~cm}^{-1}$ in the two parts of the figure.

can have significant influence on the mechanisms of nuclear spin symmetry relaxation.

The goal of the present work is thus twofold. We aim to provide an assignment and partial analysis of the $v_{2}+2 v_{3}$ subband for ${ }^{13} \mathrm{CH}_{4}$. Secondly we shall study the question of nuclear spin symmetry conservation of ${ }^{13} \mathrm{CH}_{4}$ in supersonic jet expansions. A preliminary account of the present work has been given in [53, 54]. Figure 1 shows an introductory survey of the spectrum of ${ }^{12} \mathrm{CH}_{4}$ and ${ }^{13} \mathrm{CH}_{4}$ in the icosad range.

\section{Experimental}

Details of our experimental setup for supersonic jet/cavity ring-down laser spectroscopy have been described before [47]. We thus summarize here only the main aspects. A near-infrared InGaAsP laser diode (Radians Innova) was used as light source; it emits at most $1 \mathrm{~mW}$ between about $7500 \mathrm{~cm}^{-1}$ and $8000 \mathrm{~cm}^{-1}$ in singlefrequency mode. For the measurements shown in this paper, minor parts of the laser output are diverted to a $500 \mathrm{MHz}$ external monitor etalon and a wavemeter (Burleigh, WA-1500-NIR) for relative calibration. The main part of the laser output is led through an Acousto-Optical Modulator (AOM Isomet, 1205C-2); the first- 
order deflection is transferred through a single-mode optical fiber and coupled into an optical cavity using a lens to match the geometry of the laser beam to the single transversal cavity mode $\mathrm{TEM}_{00}$. The cavity is composed of two concave mirrors with $1 \mathrm{~m}$ radius and high reflectivity $R>99.97 \%$ (Newport) mounted on an optical bench (Newport mirror holders and Spindler and Hoyer, Mikrobank) at a distance of about $33 \mathrm{~cm}$. The cavity is set up in a vacuum chamber, the residual vacuum (typically of some $10^{-7} \mathrm{mbar}$ ) being maintained by a $8000 \mathrm{l} / \mathrm{s}$ oil diffusion pump, backed by a combination of a vacuum roots blower pump and a mechanical roughing pump. The absorption path is perpendicularly crossed by a supersonic jet produced by a solenoid pulsed $40 \times 0.1 \mathrm{~mm}^{2}$-slit nozzle aligned along the optical axis of the cavity at a probe distance to the optical axis which can be adjusted up to about $1 \mathrm{~cm}$. The nozzle produces narrow gas pulses with a plateau of at least $200 \mu \mathrm{s}$, which allows for semi-quantitative measurements [47]. Under our experimental conditions, with less than 1 bar backing pressure, the dynamical pressure in the cavity does not exceed about $2 \times 10^{-4} \mathrm{mbar}$ during the expansion.

Continuous wave (cw)-laser CRD spectroscopy requires to match the cavity length to the wavelength of the laser; this can be achieved by periodically wobbling the cavity length and switching off the light entering the cavity with the use of the AOM when a resonance is observed through a maximum in the intensity of the light transmitted through the cavity [55]. The Ring-Down (RD) event, i.e. the decay of the transmitted light through the cavity as a function of time, is then measured. The current version of our timing- and trigger-scheme is described in detail in [47]. The resonances which occur while the nozzle is not activated are collected separately in our scheme to obtain the spectral baseline during the same scan and will be referred to as "background". This has the main advantage of saving time since both ring-down events with and without supersonic jet expansion are recorded in the same measurement; moreover it reduces possible sources of noise by long-term drifts of the baseline. In order to keep the residual pressure in the cavity as low as possible, the nozzle effectively operates at $10 \mathrm{~Hz}$.

The Ring-Down (RD) exponential decay of the intensity of light transmitted through the cavity $I(t)$ is analysed and fitted to estimate the ring-down constant $k_{\mathrm{RD}}=\tau_{\mathrm{RD}}^{-1}$ according to:

$$
I(t)=I(0) \exp \left(-k_{\mathrm{RD}} t\right)=I(0) \exp \left(-t / \tau_{\mathrm{RD}}\right) .
$$

We have previously shown that we can use the Three-Region Integration method for the fit, which requires significantly less mathematical steps than the Levenberg-Marquardt procedure with the same accuracy, provided that the absorption is not too strong [47], thus saving data acquisition and analysis time. The ring-down constant $k_{\mathrm{RD}}$ can finally be related to the absorbance per pass $A_{\mathrm{pp}}$ by 
the equations:

$$
\begin{aligned}
& k_{\mathrm{RD}}=\alpha c+(1-R) c / L, \\
& A_{\mathrm{pp}}=\alpha L,
\end{aligned}
$$

where $c$ is the speed of light. $R$ is essentially the reflectivity of the mirrors but includes also non resonant losses by scattering and diffraction, $L$ is the effective cavity length and $\alpha$ is the corresponding absorption coefficient. The second term on the right hand side of Equation (4) is actually the spectral baseline. In the case of a static experiment, the concentration $C$ of the probed molecule is constant and the molecular absorption cross section $\sigma$ could be obtained from $\sigma=\alpha / C$, but this does not apply to a pulsed jet expansion.

For the measurements shown here, typically 16 decays are accumulated for resonances with the supersonic expansion ("signal"), and 300 without ("background"); the two series of $k_{\mathrm{RD}}$ values are averaged separately and the difference of the two averaged $\bar{k}_{\mathrm{RD}}$ values constitutes one point of a CRD spectrum, which is then obtained by scanning the laser wavelength point by point. The wavelength is measured at each data acquisition point using a scanning Michelson interferometer with a built-in stabilized $\mathrm{He}-\mathrm{Ne}$ laser (Burleigh wavemeter) to get a first estimate of the wavenumber. For the relative wavenumber calibration, the etalon fringes of a home-built $500 \mathrm{MHz}$ etalon are recorded simultaneously and used to linearize the spectrum (the free spectral range of the etalon is known with great precision, and the frequency drift is less than about $1 \mathrm{MHz}$ per day). The relative wavenumber accuracy of spectral points within a spectrum is thus typically about $0.001 \mathrm{~cm}^{-1}$ for a large frequency range. For the spectra shown in this paper, the absolute wavenumber calibration has been performed with the FTIR spectrum, which was itself calibrated with water lines. The absolute wavenumber accuracy has been critically discussed in [47] in detail to be around $0.003 \mathrm{~cm}^{-1}$, depending mostly upon uncertainties of the calibration lines.

We also measured CRD spectra of ${ }^{13} \mathrm{CH}_{4}$ at room temperature, using the cavity described above as a cell, and in supersonic jet expansions using ${ }^{13} \mathrm{CH}_{4}$ : Ar gas mixtures with various mole fractions $x$ ranging from 0.01 up to 0.16 (we always quote the mole fraction $x\left({ }^{13} \mathrm{CH}_{4}\right)=\left[{ }^{13} \mathrm{CH}_{4}\right] /\left([\mathrm{Ar}]+\left[{ }^{13} \mathrm{CH}_{4}\right]\right)$ when we give quantitative data). In this latter case, we actually used the composition of the gas mixture to record spectra at various low temperatures. We chose not to change other gas expansion parameters (i.e. nozzle time opening, delay between nozzle opening and laser, backing pressure, and dynamical pressure in the cavity) so that the spectra were always recorded under the same conditions. The differences between two jet spectra can then be attributed to the composition of the gas mixture only. 
Table 3: Experimental conditions for the static measurements of the $\mathrm{R}(0)$ line (mole fraction $x\left({ }^{13} \mathrm{CH}_{4}\right)$ and total pressure $\left.p_{\text {tot }}\right)$ as well as the estimated integrated rovibrational line strength $G_{\mathrm{fi}}$, the Gaussian linewidth $\Delta \tilde{v}$ and the integrated band strength $G_{\mathrm{ba}}$.

\begin{tabular}{lrrrr}
\hline$x\left({ }^{13} \mathrm{CH}_{4}\right)$ & $p_{\text {tot }} / \mathrm{mbar}$ & $G_{\mathrm{fi}} / \mathrm{pm}^{2}$ & $\Delta \tilde{v} / \mathrm{cm}^{-1}$ & $G_{\mathrm{ba}} / \mathrm{pm}^{2}$ \\
\hline 0.025 & 0.5903 & $5.32 \times 10^{-7}$ & 0.02270 & $5.70 \times 10^{-5}$ \\
0.005 & 0.7217 & $5.00 \times 10^{-7}$ & 0.02295 & $5.36 \times 10^{-5}$ \\
0.005 & 0.8368 & $4.58 \times 10^{-7}$ & 0.02205 & $4.91 \times 10^{-5}$ \\
\hline
\end{tabular}

A Fourier Transform Infrared (FTIR) spectrum of ${ }^{13} \mathrm{CH}_{4}$ in helium (10\%) with a total pressure of $0.6 \mathrm{hPa}$ was also recorded at about $80 \mathrm{~K}$ in our group with the Bruker Zurich Prototype IFS125 (ZP2001) and a path length of $10 \mathrm{~m}$ [36, 37]. This spectrum is shown as a survey in Figure 1. The sample of ${ }^{13} \mathrm{CH}_{4}$ was obtained from Cambridge Isotope Laboratories with a stated purity of $>99 \%$ and argon was obtained from Pangas (stated purity of $99.9990 \%$ ). For the static measurements, pressures were obtained with a high accuracy pressure transducer of absolute type (pressure range: 1 Torr $=133.322 \mathrm{~Pa}$ ) from MKS Instruments, the precision of the pressure gauge being specified as $10^{-4}$ Torr. Because of the high sensitivity of the CRD spectroscopy, a very small quantity of methane ${ }^{13} \mathrm{CH}_{4}$ is needed to obtain a signal. Rather than trying to insert pure methane in the cavity at a very low pressure and thus obtain a large relative error in the estimation of the pressure, we preferred to use a gas mixture of argon and methane: this makes it possible to work with a higher total pressure and a higher precision of the measurements although the partial pressure of methane is low enough to prevent any saturation of the CRD signal. The total pressure and the $\left[{ }^{13} \mathrm{CH}_{4}\right] /[\mathrm{Ar}]$ ratio are such that any pressure broadening is negligible.

During the measurements of one line, we observed a slight and continuous increase of the total pressure of about $5 \%$, which is probably due either to desorption from the walls of the cavity or/and a small leak from the nozzle of the jet expansion because the cavity is not evacuated during the measurement. Nevertheless, we observed no effect on the spectrum, and we believe that it does not perturb our measurements for the estimation of the band strength. Three measurements of the $\mathrm{R}(0)$ transition were recorded with various pressures and compositions of the gas mixture and the experimental conditions are listed in Table 3. The static measurements were carried out in a temperature controlled laboratory at $T=(296 \pm 1) \mathrm{K}$. 


\section{Theoretical analysis}

Although the theoretical analysis of the spectrum and that of nuclear spin symmetry conservation and relaxation have been discussed in previous work [ $45,47,48]$ for ${ }^{12} \mathrm{CH}_{4}$, we briefly recall the basic ideas here in order to define notations and to have a self contained description, emphasizing how the concepts help us to investigate nuclear spin symmetry conservation as well as to assign the rovibrational transitions in spectra obtained at different temperatures.

\subsection{Level structure and symmetry notation}

Methane has a regular tetrahedral equilibrium structure and therefore is often described with the point group $T_{\mathrm{d}}[40]$. Vibrational overtone and combination levels are split into sublevels by the so-called tetrahedral splittings [36, 40], as the possible degeneracies are defined by the irreducible representations $A_{1}, A_{2}, E, F_{1}$, and $\mathrm{F}_{2}$ with degeneracy 1 for A-levels, 2 for E-levels, and 3 for F-levels in $T_{\mathrm{d}}$.

The icosad possesses 134 sublevels, 18 being of $A_{1}, 11$ of $A_{2}, 28$ of E, 34 of $\mathrm{F}_{1}$ and 43 of $\mathrm{F}_{2}$ symmetry (see [36, 47]). $v_{2}+2 v_{3}$ is a particularly important chromophore level with two quanta of $\mathrm{F}_{2}$-stretching and one quantum of the $\mathrm{E}$ bending mode. The representation of the 12 -fold degenerate $v_{2}+2 v_{3}$ vibrational level is reducible to 6 components, namely $\mathrm{A}_{1}, \mathrm{~A}_{2}, 2 \mathrm{E}, \mathrm{F}_{1}$ and $\mathrm{F}_{2}$ which are coupled by vibrational-rotational interactions $[45,47]$. Only the term with the $\mathrm{F}_{2}$ species results in a strongly allowed infrared transition from the ground state and its three components $\mathrm{F}^{(-)}, \mathrm{F}^{(0)}$ and $\mathrm{F}^{(+)}$are coupled by Coriolis interactions [40].

If one considers the tunneling sublevels due to the inversion of the four $\mathrm{H}$ atoms with respect to the $\mathrm{C}$ atom, the molecule has to be described with a permutation-inversion group following Longuet-Higgins [56-60]. In the following, methane will be described in the $S_{4}^{*}$ group which is the direct product of the symmetric group of permutations of four objects $S_{4}$ and the inversion group $S^{*}$; we explicitly indicate the parity of each species with + or - following the notation of $[57,58]$. The treatment is identical to that of ${ }^{12} \mathrm{CH}_{4}$ (see Reference [47]), except that the ${ }^{13} \mathrm{C}$ nucleus has a spin of $1 / 2$ and a negative parity while all nuclei in ${ }^{12} \mathrm{CH}_{4}$ have positive parity. In the following, as the assignment often refers to that of ${ }^{12} \mathrm{CH}_{4}$ for comparison, the parities are given as rovibrational parities (excluding nuclear spins) which are the same for ${ }^{12} \mathrm{CH}_{4}$ and for ${ }^{13} \mathrm{CH}_{4}$. For convenience Table 4 gives a summary of the sublevel structure for Pauli allowed rovibrational species in $\mathrm{S}_{4}^{*}$ for ${ }^{12} \mathrm{CH}_{4}$ and ${ }^{13} \mathrm{CH}_{4}$ including ${ }^{12} \mathrm{C}$ spin and ${ }^{13} \mathrm{C}$ spin where the parities are reversed for the latter because of the negative parity of the ${ }^{13} \mathrm{C}$ nucleus. 
Table 4: Summary of symmetry species of ${ }^{12} \mathrm{CH}_{4}$ and ${ }^{13} \mathrm{CH}_{4}$ in the groups $T, T_{\mathrm{d}}$ and $S_{4}^{*}$ together with the allowed total nuclear spin $I$ from the four protons (with Pauli-allowed nuclear spin symmetry species $\Gamma_{\text {ns }}$ in parentheses) and the Pauli-allowed sublevel structure, the symmetry label $\Gamma_{\mathrm{P}}$ including parity from the ${ }^{13} \mathrm{C}$ nucleus for ${ }^{13} \mathrm{CH}_{4}$. The column $\Gamma_{\mathrm{P}}\left({ }^{12} \mathrm{CH}_{4}\right)$ gives also the rovibronic species for ${ }^{13} \mathrm{CH}_{4}$ (as for ${ }^{12} \mathrm{CH}_{4}$ ) excluding ${ }^{13} \mathrm{C}$ nuclear spin, which we use in most of the present paper for simplicity because of the similarity for the two isotopomers. $[\cdot, \cdot]^{ \pm}$gives the partition in the permutation group $S_{4}$ with parity + or - in $S^{*}$ as upper index [58]. $I\left(\Gamma_{\mathrm{s}}\right)$ gives the total nuclear spin from the four protons for the symmetry species $\Gamma_{\mathrm{s}}$ of the nuclear spin function.

\begin{tabular}{lllrllllr}
\hline$\Gamma(T)$ & $\Gamma\left(T_{\mathrm{d}}\right)$ & $\Gamma\left(T_{\mathrm{d}}\right) \uparrow S_{4}^{*}$ & ${\text { [partition }]^{\text {parity }}}$ & $I\left(\Gamma_{\mathrm{ns}}\right)$ & $\Gamma_{\mathrm{P}}\left({ }^{12} \mathrm{CH}_{4}\right)$ & $g_{12}$ & $\Gamma_{\mathrm{P}}\left({ }^{13} \mathrm{CH}_{4}\right)$ & $g_{13}$ \\
\hline $\mathrm{A}$ & $\mathrm{A}_{1}$ & $\mathrm{~A}_{1}^{+}+\mathrm{A}_{2}^{-}$ & {$[4]^{+}+\left[1^{4}\right]^{-}$} & $2\left(\mathrm{~A}_{1}^{+}\right)$ & $\mathrm{A}_{2}^{-}$ & 5 & $\mathrm{~A}_{2}^{+}$ & 10 \\
$\mathrm{~A}$ & $\mathrm{~A}_{2}$ & $\mathrm{~A}_{2}^{+}+\mathrm{A}_{1}^{-}$ & {$\left[1^{4}\right]^{+}+[4]^{-}$} & $2\left(\mathrm{~A}_{1}^{+}\right)$ & $\mathrm{A}_{2}^{+}$ & 5 & $\mathrm{~A}_{2}^{-}$ & 10 \\
$\mathrm{E}$ & $\mathrm{E}$ & $\mathrm{E}^{+}+\mathrm{E}^{-}$ & {$\left[2^{2}\right]^{+}+\left[2^{2}\right]^{-}$} & $0\left(\mathrm{E}^{+}\right)$ & $\mathrm{E}^{+}+\mathrm{E}^{-}$ & 2 & $\mathrm{E}^{+}+\mathrm{E}^{-}$ & 4 \\
$\mathrm{~F}$ & $\mathrm{~F}_{1}$ & $\mathrm{~F}_{1}^{+}+\mathrm{F}_{2}$ & {$\left[2,1^{2}\right]^{+}+[3,1]^{-}$} & $1\left(\mathrm{~F}_{2}^{+}\right)$ & $\mathrm{F}_{1}^{+}$ & 3 & $\mathrm{~F}_{1}$ & 6 \\
$\mathrm{~F}$ & $\mathrm{~F}_{2}$ & $\mathrm{~F}_{2}^{+}+\mathrm{F}_{1}^{-}$ & {$[3,1]^{+}+\left[2,1^{2}\right]^{-}$} & $1\left(\mathrm{~F}_{2}^{+}\right)$ & $\mathrm{F}_{1}^{-}$ & 3 & $\mathrm{~F}_{1}^{+}$ & 6 \\
\hline
\end{tabular}

It also shows the sublevel structure $\Gamma\left(T_{\mathrm{d}}\right) \uparrow S_{4}^{*}$ of the sublevels which are nearly degenerate due to slow inversion of methane with an inversion barrier of about $400 \mathrm{~kJ} \mathrm{~mol}^{-1}[27]$ (see also $[9,10,47,58]$ ).

\subsection{Nuclear spin symmetry}

Because of the four equivalent $\mathrm{H}$ atoms with nuclear spin $I_{\mathrm{H}}=1 / 2$, methane ${ }^{13} \mathrm{CH}_{4}$, like ${ }^{12} \mathrm{CH}_{4}$, has three nuclear spin isomers (with total nuclear spin from the protons $I=0,1$ or 2). The representation of the corresponding 16 nuclear spin functions is reducible to ${ }^{5} \mathrm{~A}_{1}^{+}(I=2), \mathrm{E}^{+}(I=0)$ and ${ }^{3} \mathrm{~F}_{2}^{+}(I=1)$ components in the $S_{4}^{*}$ permutation-inversion group, where the upper left index indicates the frequency of that species in the reduction, and the multiplicity due to nuclear spin. The so-called meta nuclear spin isomer $(I=2)$ corresponds to the symmetry $\Gamma_{\mathrm{ns}}=\mathrm{A}_{1}^{+}$, ortho $(I=1)$ to the symmetry $\Gamma_{\mathrm{ns}}=\mathrm{F}_{2}^{+}$and para $(I=0)$ to the symmetry $\Gamma_{\mathrm{ns}}=\mathrm{E}^{+}$. According to the generalized Pauli principle, only the nurovibrational states that transform as $\mathrm{A}_{2}^{ \pm}$are allowed [58] (the terminology nurovibrational is used for nuclear spin-rotational and vibrational states); as a consequence the rovibrational states of the meta nuclear spin isomer transform as $\mathrm{A}_{2}^{ \pm}$, those of the ortho nuclear spin isomer transform as $\mathrm{F}_{1}^{ \pm}$and those of the para nuclear spin isomer transform as $\mathrm{E}^{ \pm}$, the electronic and vibrational ground states of methane being totally symmetric $\left(\mathrm{A}_{1}\right.$ in $\left.T_{\mathrm{d}}\right)$. In order to obtain total nuclear spin including the spin of ${ }^{13} \mathrm{C}$ one has to combine the total hydrogenic spin $I$ (from the four protons) with $I\left({ }^{13} \mathrm{C}\right)=1 / 2$ and reverse parities because of the negative parity of ${ }^{13} \mathrm{C}$. 
The hyperfine effects from ${ }^{13} \mathrm{C}$ are, however, not revealed in our spectra. The column $I\left(\Gamma_{\mathrm{ns}}\right)$ in Table 4 indicates which nuclear spin $I$ with nuclear spin symmetry $\Gamma_{\mathrm{ns}}$ combines with the corresponding rovibrational sublevels.

In the case of nuclear spin symmetry conservation (indexed "c") during an experiment involving a cooling process (in our case, either in a collisional cooling cell or in a supersonic jet expansion), the three nuclear spin isomers keep their relative population set before the experiment at room temperature. One observes a superposition of Boltzmann distributions, each within the rovibrational levels of a given nuclear spin isomer. Hence, the relative population of the $n$-th rotational level with given $J$ of the nuclear spin isomer $\Gamma_{\mathrm{ns}}$ (in order of increasing energy) at rotational temperature $T_{\text {rot }}$ can be written as follows:

$$
p^{\mathrm{c}}\left(J, n, \Gamma_{\mathrm{ns}}, T_{0}, T_{\mathrm{rot}}\right)=\frac{x\left(\Gamma_{\mathrm{ns}}, T_{0}\right)(2 J+1)}{\mathrm{Q}_{\mathrm{rot}}\left(\Gamma_{\mathrm{ns}}, T_{\mathrm{rot}}\right)} \exp \left(-\frac{E_{n}\left(J, \Gamma_{\mathrm{ns}}\right)-E_{0}\left(\Gamma_{\mathrm{ns}}\right)}{k_{\mathrm{B}} T_{\mathrm{rot}}}\right),
$$

where $x\left(\Gamma_{\mathrm{ns}}, T_{0}\right)$ is the mole fraction of nuclear spin isomer $\Gamma_{\mathrm{ns}}$ at temperature $T_{0}$, at which the isomers are assumed to be equilibrated:

$$
x\left(\Gamma_{\mathrm{ns}}, T_{0}\right)=g\left(\Gamma_{\mathrm{ns}}\right) \frac{Q_{\mathrm{rot}}\left(\Gamma_{\mathrm{ns}}, T_{0}\right)}{\mathrm{Q}_{\mathrm{rot}}^{\mathrm{r}}\left(T_{0}\right)} \exp \left(-\frac{E_{0}\left(\Gamma_{\mathrm{ns}}\right)}{k_{\mathrm{B}} T_{0}}\right) .
$$

$E_{0}\left(\Gamma_{\mathrm{ns}}\right)$ is the energy of the lowest level of nuclear spin isomer $\Gamma_{\mathrm{ns}}$ and $E_{n}\left(J, \Gamma_{\mathrm{ns}}\right)$ that of the $n$-th level with angular momentum quantum number $J . g\left(\Gamma_{\mathrm{ns}}\right)$ is the nuclear spin statistical weight factor; in our case, $g\left(\mathrm{~A}_{1}^{+}\right)=5, g\left(\mathrm{~F}_{1}^{+}\right)=3$, and $g\left(\mathrm{E}^{ \pm}\right)=2$. The rotational partition function $Q_{\text {rot }}\left(\Gamma_{\mathrm{ns}}, T\right)$ of nuclear spin isomer $\Gamma_{\mathrm{ns}}$ at temperature $T$ in Equations (6) and (7) is defined as follows:

$$
Q_{\text {rot }}\left(\Gamma_{\mathrm{ns}}, T\right)=\sum_{J}(2 J+1)\left[\sum_{n} \exp \left(-\frac{E_{n}\left(J, \Gamma_{\mathrm{ns}}\right)-E_{0}\left(\Gamma_{\mathrm{ns}}\right)}{k_{\mathrm{B}} T}\right)\right] .
$$

In Equation (7), $Q_{\mathrm{rot}}^{\mathrm{r}}(T)$ is the relaxed rotational partition function including nuclear spin at temperature $T$ and is defined as follows:

$$
Q_{\mathrm{rot}}^{\mathrm{r}}(T)=\sum_{\Gamma_{\mathrm{ns}}} g\left(\Gamma_{\mathrm{ns}}\right) Q_{\mathrm{rot}}\left(\Gamma_{\mathrm{ns}}, T\right) \exp \left(-\frac{E_{0}\left(\Gamma_{\mathrm{ns}}\right)}{k_{\mathrm{B}} T}\right) .
$$

In the case of nuclear spin symmetry relaxation (indexed "r") during a cooling process, the nuclear spin states are allowed to change via collisions; hence the relative populations of the rotational levels should represent a global thermal equilibrium among all states, including low temperatures. In this case, the relative population of the $n$-th rotational state with angular momentum quantum number $J$ of the nuclear spin isomer $\Gamma_{\mathrm{ns}}$ at rotational temperature $T_{\text {rot }}$ can be written as follows:

$$
p^{\mathrm{r}}\left(J, n, \Gamma_{\mathrm{ns}}, T_{\mathrm{rot}}\right)=\frac{g\left(\Gamma_{\mathrm{ns}}\right)(2 J+1)}{Q_{\mathrm{rot}}^{\mathrm{r}}\left(T_{\mathrm{rot}}\right)} \exp \left(-\frac{E_{n}\left(J, \Gamma_{\mathrm{ns}}\right)}{k_{\mathrm{B}} T_{\mathrm{rot}}}\right) .
$$


The intramolecular mechanism usually assumed for nuclear spin symmetry relaxation in methane and other small molecules is the so-called quantum relaxation model [51, 52]: it assumes the existence of close lying states of the different nuclear spin isomers that are coupled and can be populated via collisions. This leads to rather fast nuclear spin symmetry relaxation in bulk samples at room temperature on time scales of seconds to hours in polyatomic molecules. However, the number of collisions during a supersonic expansion is not expected to be sufficient to allow for thermal equilibrium between the different nuclear spin isomers [45, 47-52, 57, 58, 61, 62]. Experiments on the nuclear spin isomerization of methane ${ }^{12} \mathrm{CH}_{4}[63,64]$, as well as measurements in a supersonic expansion $[45,47-49,61]$ or at low temperatures with a collisional cooling technique $[37,50]$ have confirmed the conservation of nuclear spin symmetry of ${ }^{12} \mathrm{CH}_{4}$ in supersonic jet expansions.

Possible nuclear spin symmetry conservation or relaxation can be investigated spectroscopically. Indeed, the effective integrated absorption cross section $G^{\text {eff }}\left(J^{\prime}, \Gamma^{\prime}, n^{\prime} \leftarrow J, \Gamma, n, T_{\text {rot }}\right)$ of the rovibrational line corresponding to the transition from the $n$-th level with angular momentum quantum number $J$ of the rovibrational species $\Gamma$ in the lower vibrational state to the $n^{\prime}$-th level characterized by $J^{\prime}$ of the rovibrational species $\Gamma^{\prime}$ in the upper vibrational state is defined as follows:

$$
\begin{aligned}
G^{\mathrm{eff}}\left(J^{\prime}, n^{\prime}, \Gamma^{\prime} \leftarrow J, n, \Gamma, T_{\text {rot }}\right) & =\int_{\text {line }} \alpha\left(T_{\text {rot }}, \tilde{v}\right) \tilde{v}^{-1} \mathrm{~d} \tilde{v}=C \times \int_{\text {line }} \sigma\left(T_{\text {rot }}, \tilde{v}\right) \tilde{v}^{-1} \mathrm{~d} \tilde{v} \\
& =C \times G\left(J^{\prime}, n^{\prime}, \Gamma^{\prime}, \leftarrow J, n, \Gamma, T_{\text {rot }}\right),
\end{aligned}
$$

where $\alpha\left(T_{\text {rot }}, \tilde{v}\right)$ is the absorption coefficient at temperature $T_{\text {rot }}$ and wavenumber $\tilde{v}, \sigma\left(T_{\text {rot }}, \tilde{v}\right)$ the corresponding absorption cross section and $C$ the density of the absorbing species. As already discussed in previous work from our group $[37,45,47,48,50]$, the integrated absorption cross section $G\left(J^{\prime}, n^{\prime}, \Gamma^{\prime} \leftarrow\right.$ $J, n, \Gamma, T_{\text {rot }}$ ) depends on the rotational temperature and can be related approximately to the relative population of the initial state as follows:

$$
G\left(J^{\prime}, n^{\prime}, \Gamma^{\prime} \leftarrow J, n, \Gamma, T_{\text {rot }}\right) \propto p\left(J, n, \Gamma, T_{\text {rot }}\right) A\left(J, J^{\prime}\right),
$$

where $A\left(J, J^{\prime}\right)$ is the Hönl-London factor for a $J^{\prime} \leftarrow J$ transition. In the case of a spherical top molecule, the Hönl-London factor is given by:

$$
A\left(J, J^{\prime}\right)=\frac{2 J^{\prime}+1}{2 J+1} .
$$

Expressions using more accurate rotational line strengths are available in [37]. We have checked that this modifies the relative intensity by less than $8 \%$ regarding the 
Table 5: Lowest rotational energy levels of ${ }^{13} \mathrm{CH}_{4}$ up to $J=10$. The symmetry of the rotational species are indicated for the $T_{\mathrm{d}}$ point group and for the $S_{4}^{*}$ permutation-inversion group. The parities of the levels are given as rovibrational parities as for ${ }^{12} \mathrm{CH}_{4}$. For ${ }^{13} \mathrm{CH}_{4}$ all parities must be reversed $(+\leftrightarrow-)$ in order to obtain total parities including the ${ }^{13} \mathrm{C}$ nuclear spin function. The splittings of $\mathrm{E}^{+}$and $\mathrm{E}^{-}$levels are not resolved at the present level (estimated to be on the order of a few $\mathrm{kHz}$ for ${ }^{12} \mathrm{CH}_{4}$ in [64]). Thus we give one term value $\mathrm{E}^{ \pm}$. The levels were calculated by Niederer [37] using the XTDS program package by Wenger et al. [75].

\begin{tabular}{|c|c|c|c|}
\hline$J$ & $\Gamma_{\text {rot }}\left(T_{\mathrm{d}}\right)$ & $\Gamma_{\text {rot }}\left(S_{4}^{*}\right)$ & $E^{\prime \prime} /\left(h c \mathrm{~cm}^{-1}\right)$ \\
\hline 0 & $A_{1}$ & $\mathrm{~A}_{2}^{-}$ & 0.0000000 \\
\hline 1 & $\mathrm{~F}_{1}$ & $\mathrm{~F}_{1}^{+}$ & 10.482132 \\
\hline 2 & $\mathrm{E}$ & $\mathrm{E}^{ \pm}$ & 31.443575 \\
\hline 2 & $\mathrm{~F}_{2}$ & $\mathrm{~F}_{1}^{-}$ & 31.443841 \\
\hline 3 & $\mathrm{~F}_{1}$ & $\mathrm{~F}_{1}^{+}$ & 62.878689 \\
\hline 3 & $\mathrm{~F}_{2}$ & $\mathrm{~F}_{1}$ & 62.879752 \\
\hline 3 & $\mathrm{~A}_{2}$ & $\mathrm{~A}_{2}^{+}$ & 62.881080 \\
\hline 4 & $A_{1}$ & $\mathrm{~A}_{2}^{-}$ & 104.777699 \\
\hline 4 & $\mathrm{~F}_{1}$ & $\mathrm{~F}_{1}^{+}$ & 104.779560 \\
\hline 4 & $\mathrm{E}$ & $\mathrm{E}^{ \pm}$ & 104.780889 \\
\hline 4 & $\mathrm{~F}_{2}$ & $\mathrm{~F}_{1}^{-}$ & 104.784868 \\
\hline 5 & $F_{1}$ & $\mathrm{~F}_{1}^{+}$ & 157.131630 \\
\hline 5 & $\mathrm{~F}_{2}$ & $\mathrm{~F}_{1}^{-}$ & 157.135220 \\
\hline 5 & $\mathrm{E}$ & $\mathrm{E}^{ \pm}$ & 157.144490 \\
\hline 5 & $F_{1}$ & $\mathrm{~F}_{1}^{+}$ & 157.146215 \\
\hline 6 & $\mathrm{E}$ & $\mathrm{E}^{ \pm}$ & 219.923687 \\
\hline 6 & $\mathrm{~F}_{2}$ & $\mathrm{~F}_{1}^{-}$ & 219.925273 \\
\hline 6 & $\mathrm{~A}_{2}$ & $\mathrm{~A}_{2}^{+}$ & 219.930080 \\
\hline 6 & $\mathrm{~F}_{2}$ & $\mathrm{~F}_{1}^{-}$ & 219.946995 \\
\hline 6 & $\mathrm{~F}_{1}$ & $\mathrm{~F}_{1}^{+}$ & 219.951493 \\
\hline 6 & $A_{1}$ & $\mathrm{~A}_{2}^{-}$ & 219.955465 \\
\hline 7 & $\mathrm{~F}_{1}$ & $\mathrm{~F}_{1}^{+}$ & 293.136639 \\
\hline 7 & $F_{2}$ & $\mathrm{~F}_{1}^{-}$ & 293.140201 \\
\hline 7 & $\mathrm{~A}_{2}$ & $\mathrm{~A}_{2}^{+}$ & 293.167856 \\
\hline 7 & $\mathrm{~F}_{2}$ & $\mathrm{~F}_{1}^{-}$ & 293.178231 \\
\hline 7 & $\mathrm{E}$ & $\mathrm{E}^{ \pm}$ & 293.183799 \\
\hline 7 & $\mathrm{~F}_{1}$ & $\mathrm{~F}_{1}^{+}$ & 293.192345 \\
\hline 8 & $A_{1}$ & $\mathrm{~A}_{2}^{-}$ & 376.748016 \\
\hline 8 & $\mathrm{~F}_{1}$ & $\mathrm{~F}_{1}^{+}$ & 376.751300 \\
\hline 8 & $\mathrm{E}$ & $\mathrm{E}^{ \pm}$ & 376.753228 \\
\hline 8 & $\mathrm{~F}_{2}$ & $\mathrm{~F}_{1}$ & 376.803467 \\
\hline 8 & $\mathrm{~F}_{1}$ & $\mathrm{~F}_{1}^{+}$ & 376.822379 \\
\hline 8 & $\mathrm{E}$ & $\mathrm{E}^{ \pm}$ & 376.838894 \\
\hline 8 & $\mathrm{~F}_{2}$ & $\mathrm{~F}_{1}^{-}$ & 376.843875 \\
\hline 9 & $\mathrm{~F}_{1}$ & $\mathrm{~F}_{1}^{+}$ & 470.738969 \\
\hline 9 & $\mathrm{~F}_{2}$ & $\mathrm{~F}_{1}$ & 470.742355 \\
\hline
\end{tabular}


Table 5: Continued.

\begin{tabular}{lrrr}
\hline$J$ & $\Gamma_{\text {rot }}\left(T_{\mathrm{d}}\right)$ & $\Gamma_{\text {rot }}\left(S_{4}^{*}\right)$ & $E^{\prime \prime} /\left(h c \mathrm{~cm}^{-1}\right)$ \\
\hline 9 & $\mathrm{E}$ & $\mathrm{E}^{ \pm}$ & 470.821004 \\
9 & $\mathrm{~F}_{1}$ & $\mathrm{~F}_{1}^{+}$ & 470.827314 \\
9 & $\mathrm{~A}_{1}$ & $\mathrm{~A}_{2}^{-}$ & 470.853009 \\
9 & $\mathrm{~F}_{1}$ & $\mathrm{~F}_{1}^{+}$ & 470.877051 \\
9 & $\mathrm{~F}_{2}$ & $\mathrm{~F}_{1}^{-}$ & 470.887112 \\
9 & $\mathrm{~A}_{2}$ & $\mathrm{~A}_{2}^{+}$ & 470.894973 \\
10 & $\mathrm{E}$ & $\mathrm{E}^{ \pm}$ & 575.078213 \\
10 & $\mathrm{~F}_{2}$ & $\mathrm{~F}_{1}^{-}$ & 575.079604 \\
10 & $\mathrm{~A}_{2}$ & $\mathrm{~A}_{2}^{+}$ & 575.082619 \\
10 & $\mathrm{~F}_{2}$ & $\mathrm{~F}_{1}^{-}$ & 575.197050 \\
10 & $\mathrm{~F}_{1}$ & $\mathrm{~F}_{1}^{+}$ & 575.211285 \\
10 & $\mathrm{~A}_{1}$ & $\mathrm{~A}_{2}^{-}$ & 575.249909 \\
10 & $\mathrm{~F}_{1}$ & $\mathrm{~F}_{1}^{+}$ & 575.286792 \\
10 & $\mathrm{E}_{1}$ & $\mathrm{E}^{ \pm}$ & 575.298926 \\
10 & $\mathrm{~F}_{2}$ & $\mathrm{~F}_{1}^{-}$ & 575.312430 \\
\hline
\end{tabular}

various components of a given $J$ value and by less than $4 \%$ in between $J$ values, which is not important for our analysis here.

Table 5 lists the rotational levels of the vibrational ground state of methane ${ }^{13} \mathrm{CH}_{4}$ (up to $J=10$ ) that were used in our calculations to estimate the relative populations $p\left(J, n, \Gamma_{\mathrm{ns}}, T_{\text {rot }}\right)$ for rotational levels with $J \leq 4$. The contribution of rotational levels with higher $J$ values is negligible according to our calculations; furthermore transitions involving these levels clearly exhibit strong perturbation due to couplings, preventing a simple assignment. Figure 2 shows the changes of the relative populations of the first 11 rotational levels up to $J=4$ as a function of the rotational temperature in the case of nuclear spin symmetry conservation (with $T=300 \mathrm{~K}$ no longer mentioned in the notations to simplify the expression) and nuclear spin symmetry relaxation. Since for $J \leq 4$ there is only one level per nuclear spin isomer, parity and $J$ value, the value $n=1$ will not be mentioned here in the notations from now on. Also, since each rovibrational species is uniquely associated with a particular nuclear spin isomer, only the rovibrational species are indicated to retain the spectroscopic information. The splittings between $\mathrm{E}^{+}$ and $\mathrm{E}^{-}$sublevels are not resolved at the precision of Table 5 . For the $\mathrm{F}_{1}^{+}$and $\mathrm{F}_{1}^{-}$ sublevels of a given $J$, the splittings are resolved at $J=3$ and $J=4$ contrary to $\mathrm{E}^{+}$and $\mathrm{E}^{-}$levels, but the difference between the two is not observable in Figure 2, the functions representing the population for each of the two sublevels. Considering the relative populations of the 11 lowest rotational levels up to $J=4$ shown in Figure 2, in principle, one might determine if nuclear spin symmetry relaxation 


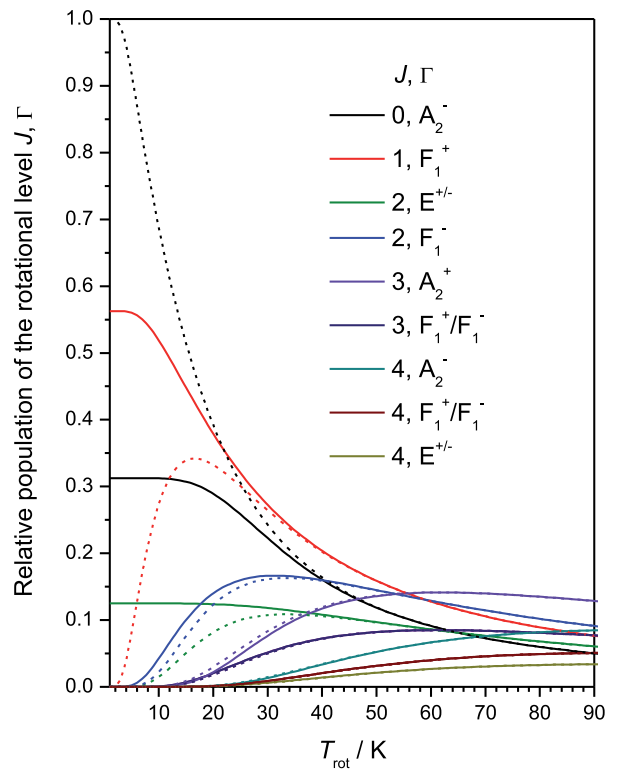

Figure 2: Relative population of the lowest 11 rotational levels of ${ }^{13} \mathrm{CH}_{4}$ with $0 \leq J \leq 4$ as a function of rotational temperature $T_{\text {rot }}$ in the case of nuclear spin symmetry conservation (full line) or relaxation (dotted line). The symmetry of the irreducible representation $\Gamma$ is indicated for the $S_{4}^{*}$ permutation-inversion group. The parities of the levels are given as rovibrational parities excluding nuclear spin. Because of the negative parity of the ${ }^{13} \mathrm{C}$ nucleus, for ${ }^{13} \mathrm{CH}_{4}$ all parities indicated here must be reversed $(+\leftrightarrow-)$ to obtain the total parities. The curves referred to as $3, \mathrm{~F}_{1}^{+} / \mathrm{F}_{1}^{-}$and $4, \mathrm{~F}_{1}^{+} / \mathrm{F}_{1}^{-}$ represent the relative population for each of the $3, \mathrm{~F}_{1}^{+}$and 3 , $\mathrm{F}_{1}^{-}$levels, respectively for each of the $4, \mathrm{~F}_{1}^{+}$and $4, \mathrm{~F}_{1}$ levels.

occurs already at $T_{\text {rot }} \leq 40 \mathrm{~K}$. However for $T_{\text {rot }}<20 \mathrm{~K}$, one should be able to unambiguously determine if it occurs (or to what extent) taking the sensitivity of the experimental setup into account.

The absolute concentration of absorbing species $C$ is not accurately known in a supersonic jet expansion. However, several measurements in our group have shown that the expansion can be considered as uniform during a day of measurements and thus the relative concentration $C$ is assumed to be effectively constant $[45,47,65,66]$. Therefore, in the following we will consider the relative effective integrated absorption cross sections (or $G$-ratios) instead of the effective integrated absorption cross sections themselves:

$$
\frac{G^{\mathrm{eff}}\left(J_{1}^{\prime}, \Gamma_{1}^{\prime} \leftarrow J_{1}, \Gamma_{1}, T_{\mathrm{rot}}\right)}{G^{\mathrm{eff}}\left(J_{2}^{\prime}, \Gamma_{2}^{\prime} \leftarrow J_{2}, \Gamma_{2}, T_{\mathrm{rot}}\right)}=\frac{p\left(J_{1}, \Gamma_{\mathrm{ns} 1}, T_{\mathrm{rot}}\right)}{p\left(J_{2}, \Gamma_{\mathrm{ns} 2}, T_{\mathrm{rot}}\right)} \frac{A\left(J_{1}, J_{1}^{\prime}\right)}{A\left(J_{2}, J_{2}^{\prime}\right)} .
$$

\subsection{Selection rules and rovibrational analysis of the $v_{2}+2 v_{3}$ combination band}

Regarding radiative electric dipole transitions, the nuclear spin symmetry is conserved $\left(\Gamma_{\mathrm{ns}}^{\prime}=\Gamma_{\mathrm{ns}}^{\prime \prime}\right)$ but the parity changes $(+\leftrightarrow-)$ which leads to the following 
allowed rovibrational transitions for methane in the $S_{4}^{*}$ group:

$$
\mathrm{A}_{2}^{+} \leftrightarrow \mathrm{A}_{2}^{-}, \mathrm{E}^{+} \leftrightarrow \mathrm{E}^{-} \text {, and } \mathrm{F}_{1}^{+} \leftrightarrow \mathrm{F}_{1}^{-} .
$$

In addition, the selection rules for one-photon transitions are:

$$
\Delta J=J^{\prime}-J^{\prime \prime}=0, \pm 1 \text { and } J^{\prime}=0 \leftrightarrow J^{\prime \prime}=0,
$$

which defines the three $\mathrm{P}(\Delta J=-1), \mathrm{Q}(\Delta J=0)$ and $\mathrm{R}(\Delta J=+1)$ branches corresponding to transitions to the $\mathrm{F}^{(+)}, \mathrm{F}^{(0)}$ and $\mathrm{F}^{(-)}$components. If one neglects the tetrahedral splittings, the rotational term values of an $\mathrm{F}_{2}$ vibrational level can be roughly approximated by:

$$
\begin{aligned}
& F^{(+)}(J)=\tilde{B}_{v} J(J+1)+\tilde{D}_{v} J^{2}(J+1)^{2}+2 \tilde{B}_{v} \zeta_{v} J, \\
& F^{(0)}(J)=\tilde{B}_{v} J(J+1)+\tilde{D}_{v} J^{2}(J+1)^{2}, \\
& F^{(-)}(J)=\tilde{B}_{v} J(J+1)+\tilde{D}_{v} J^{2}(J+1)^{2}-2 \tilde{B}_{v} \zeta_{v}(J+1),
\end{aligned}
$$

where $\tilde{B}_{v}$ is the effective rotational constant of the vibrational state $v, \tilde{D}_{v}$ is the centrifugal distortion constant to the first order correction and $\zeta_{v}$ is the Coriolis coupling constant $[40,67]$. The symbol $\sim$ above the rotational constants indicates that they are given as wavenumbers $\left(\mathrm{in}^{-1} \mathrm{~cm}^{-}\right.$.

Following Moret-Bailly [67], the equations for an effective "isolated band" fit of the rotational lines corresponding to the $\mathrm{F}_{2}$ chromophore of $v_{2}+2 v_{3}$ are then:

$$
\begin{aligned}
& \tilde{v}[\mathrm{P}(J, \Gamma)]=\tilde{v}_{0}+F^{(+)}(J-1, \Gamma)-\tilde{E}^{\prime \prime}(J, \Gamma)=a_{J} \times(-1)^{J} F_{\mathrm{A}_{1} \Gamma \Gamma}^{4 J}+b_{J}, \\
& \tilde{v}[\mathrm{Q}(J, \Gamma)]=\tilde{v}_{0}+F^{(0)}(J, \Gamma)-\tilde{E}^{\prime \prime}(J, \Gamma)=e_{J} \times(-1)^{J} F_{\mathrm{A}_{1} \Gamma \Gamma}^{4 J}+f_{J}, \\
& \tilde{v}[\mathrm{R}(J, \Gamma)]=\tilde{v}_{0}+F^{(-)}(J+1, \Gamma)-\tilde{E}^{\prime \prime}(J, \Gamma)=c_{J} \times(-1)^{J} F_{\mathrm{A}_{1} \Gamma \Gamma}^{4 J J}+d_{J} .
\end{aligned}
$$

The $F_{\mathrm{A}_{1} \Gamma \Gamma}^{4 J J}$ are the Clebsch-Gordan coefficients as given in reference [68]. $\tilde{v}_{0}$ is the band center of the excited vibrational state; it can be directly determined from the $\mathrm{P}(1)$ transition to the lowest quantum level $\mathrm{F}^{(+)}(0)$ of the $v_{2}+2 v_{3}$ vibrational state by adding the rovibrational energy for the $J=1$ ground state level to the transition wavenumber. In Equations (20)-(22), $\tilde{E}^{\prime \prime}(J, \Gamma)$ is the energy of the $J$-th level of symmetry $\Gamma$ in the vibrational ground state as indicated in Table 5. Following the notations and conventions given by Moret-Bailly [67], the parameters $a_{J}, b_{J}, c_{J}, d_{J}$, $e_{J}$, and $f_{J}$ in Equations (20)-(22) are linear combinations of the effective spectroscopic constants given in Equations (17)-(19). Therefore, a linear fit of the experimentally measured transitions of various branches for low $J$ values as a function of $(-1)^{J} F_{\mathrm{A}_{1} \Gamma \Gamma}^{4 J J}$ allows us to get an estimation of the spectroscopic constants (see also [47] for more details). We stress that we use here a very approximate analysis only for a quasi-isolated level. A more complete analysis of the interacting icosad levels would be possible using more complete effective hamiltonians [72] and more experimental data (see also the discussion in [36]). 


\section{Results}

Figure 1 shows an overview of the FTIR spectrum of methane ${ }^{13} \mathrm{CH}_{4}$ recorded at about $80 \mathrm{~K}$ in the region of the $v_{2}+2 v_{3}$ combination band. The corresponding FTIR spectrum of ${ }^{12} \mathrm{CH}_{4}$ is shown for comparison. Obviously, the two spectra globally exhibit similarities, except for the shift of the absolute wavenumber due to the isotope effect. The similarities and differences will be discussed and used in the following for the assignment of the transitions. Figure 3 shows a schematic survey of the energy levels and transitions observed in the present work.
P branch
$\mathrm{F}^{(+)}$component
Q branch
$\mathrm{F}^{(0)}$ component
R branch
$\mathrm{F}^{(-)}$component

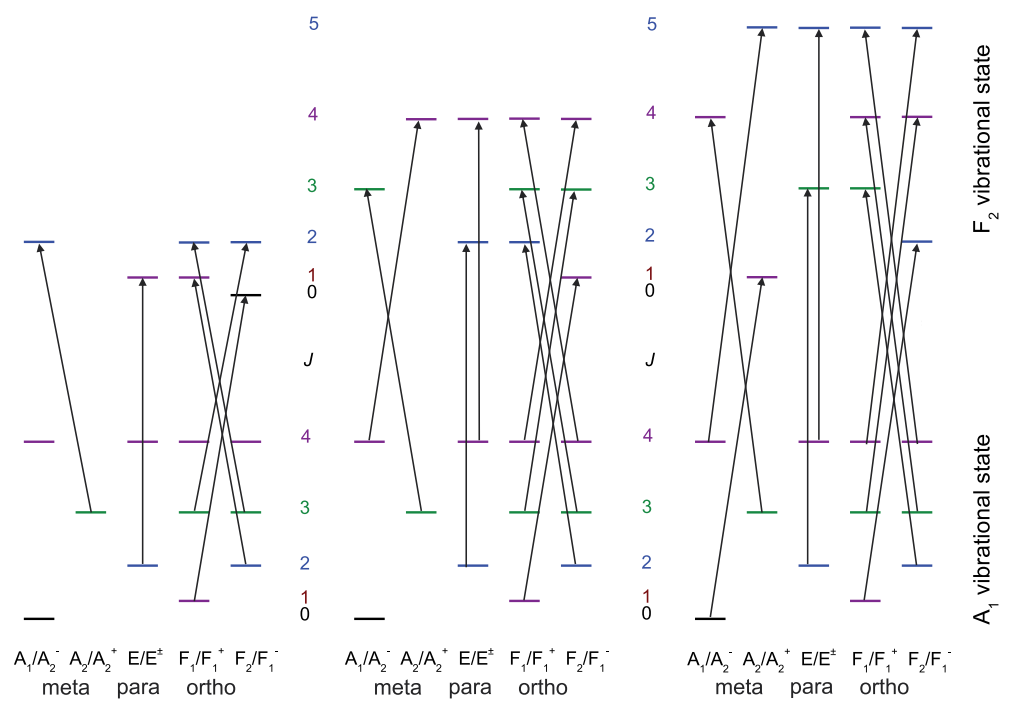

Figure 3: Energy level diagram of ${ }^{13} \mathrm{CH}_{4}$ and rovibrational transitions investigated in this work between the $A_{1}$ vibrational ground state and the $F^{(+)}, F^{(0)}$ and $F^{(-)}$components of the $F_{2}$ vibrational level $\left(v_{2}+2 v_{3}\right)$ are indicated together with their symmetry in the $T_{\mathrm{d}} / S_{4}^{*}$ group. The parities of the levels are given as rovibrational parities excluding nuclear spin. Because of the negative parity of the ${ }^{13} \mathrm{C}$ nucleus, for ${ }^{13} \mathrm{CH}_{4}$ all parities indicated here must be reversed (+ $\leftrightarrow$ ) to obtain the total parities. 

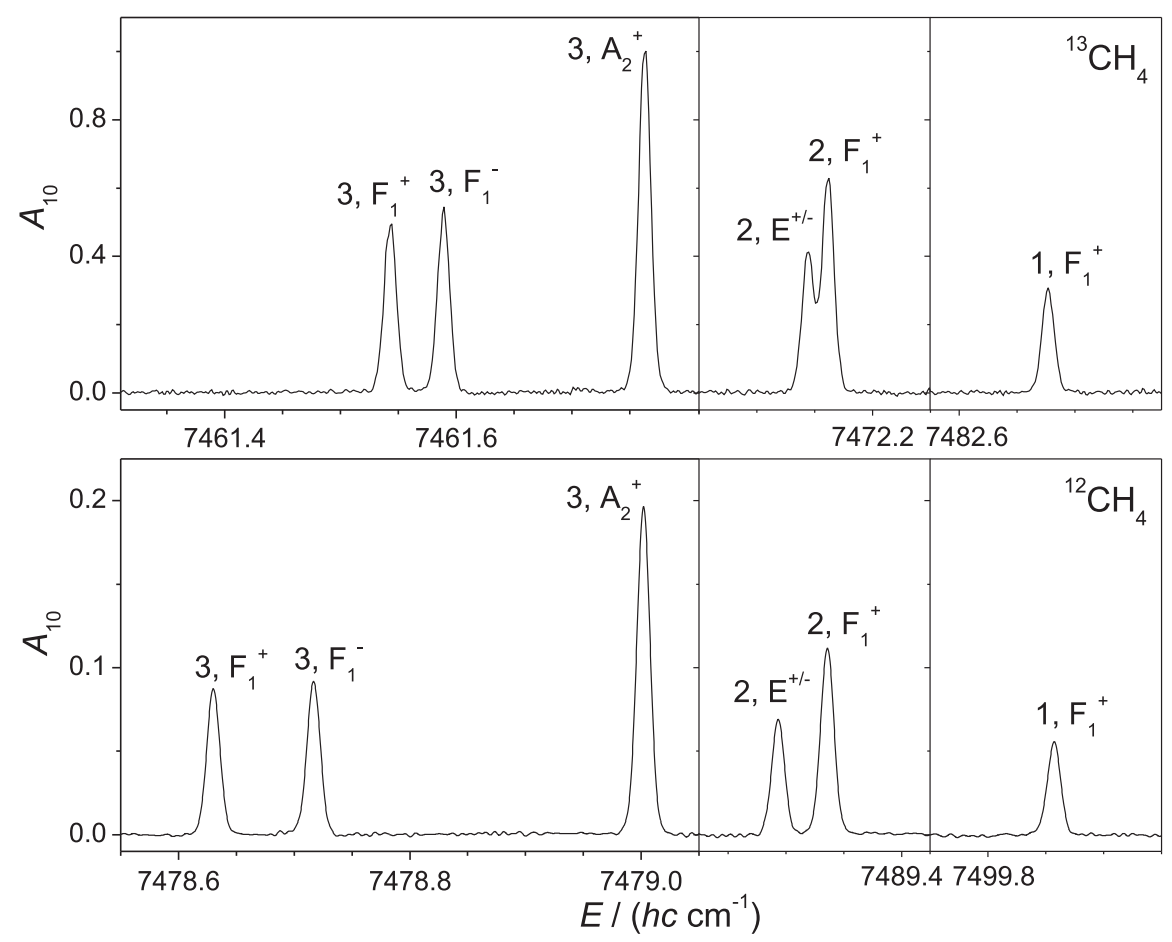

Figure 4: FTIR spectrum of the lowest components of the $\mathrm{P}$ branch of the $v_{2}+2 v_{3}$ combination band of methane ${ }^{13} \mathrm{CH}_{4}$ (top) and ${ }^{12} \mathrm{CH}_{4}$ (bottom) for comparison. The experimental conditions are as in Figure 1. The assignment for ${ }^{12} \mathrm{CH}_{4}$ is indicated in the bottom spectrum in the form of the lower state of the transition according to Reference [47]. Both spectra were recorded at $80 \mathrm{~K}$ and are shown in decadic absorbance $A_{10}=\log _{10}\left(I_{0} / I\right)$ (see Reference [36]).

\subsection{Analysis of the P and R branches, band center and band strength}

Figures 4 and 5 show the FTIR spectrum of ${ }^{13} \mathrm{CH}_{4}$ in the region of the $\mathrm{P}$ and $\mathrm{R}$ branches of the $v_{2}+2 v_{3}$ combination band recorded at $80 \mathrm{~K}$ with the Zurich Bruker Prototype ZP2001, together with that of ${ }^{12} \mathrm{CH}_{4}$ for comparison [36]. The two spectra were recorded with similar experimental conditions, except for the pressures of the molecule probed, which explains the difference in absorbance. Without taking the absolute position of the lines into account, the striking point is that the two spectra look quite similar in both $\mathrm{P}$ and $\mathrm{R}$ branches. Moreover, since the $J$ components are quite well separated from each other, based on our work for ${ }^{12} \mathrm{CH}_{4}$, the assignment of the transitions is straightforward. Table 6 shows the assignment of the $\mathrm{P}$ branch up to $J^{\prime \prime}=3$ and that of the R branch up to $J^{\prime \prime}=4$. Higher 


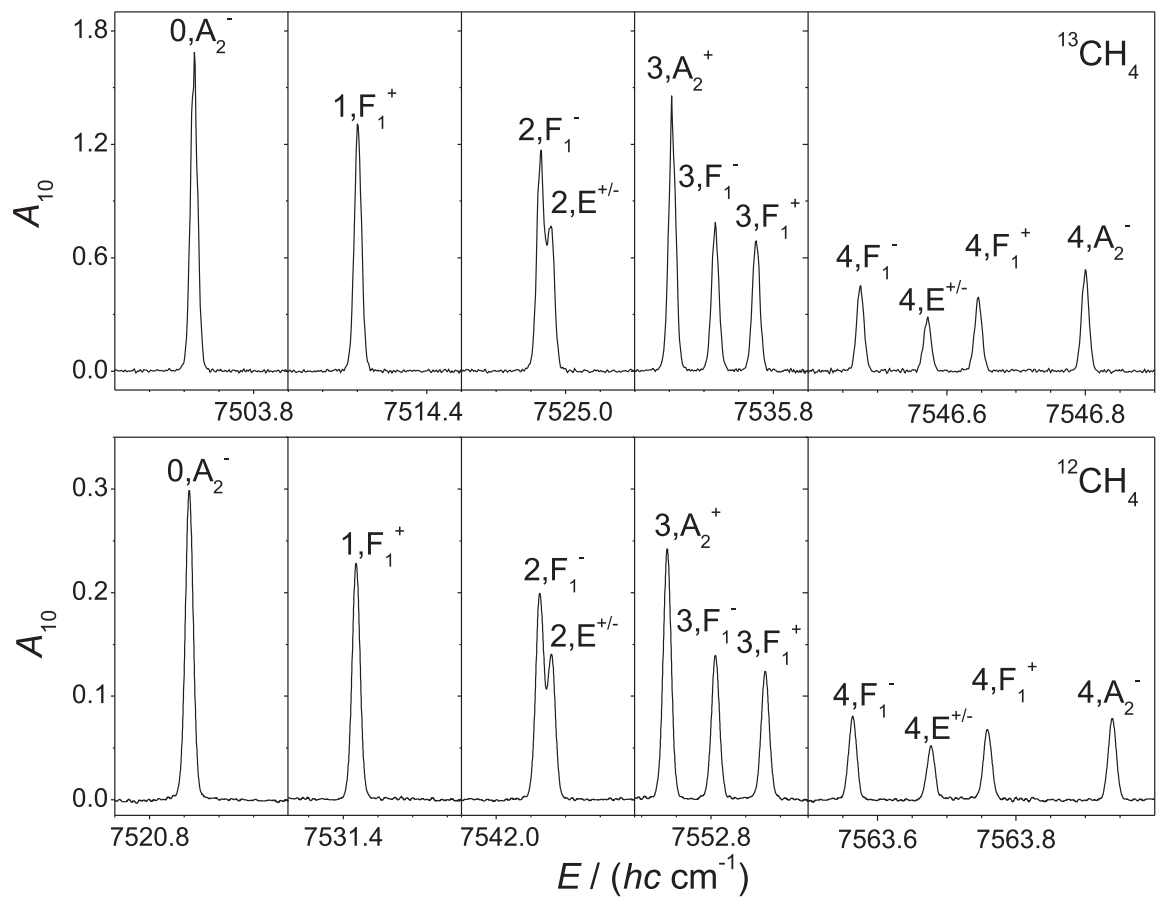

Figure 5: FTIR spectrum of the lowest components of the R branch of the $v_{2}+2 v_{3}$ combination band of methane ${ }^{13} \mathrm{CH}_{4}$ (top) and ${ }^{12} \mathrm{CH}_{4}$ (bottom) for comparison. The experimental conditions are as in Figure 1. The assignment for ${ }^{12} \mathrm{CH}_{4}$ is indicated in the bottom spectrum in the form of the lower state of the transition according to Reference [47]. Both spectra were recorded at $80 \mathrm{~K}$ and are shown in decadic absorbance $A_{10}=\log _{10}\left(I_{0} / I\right)$ (see Reference [36]).

components show perturbations that prevent further simple assignments as was also the case for ${ }^{12} \mathrm{CH}_{4}[45,47]$. From the $\mathrm{P}\left(1, \mathrm{~F}_{1}^{+}\right)$transition at $7482.67705 \mathrm{~cm}^{-1}$, we obtain a result for the band center, $\tilde{v}_{0}=7493.15918 \mathrm{~cm}^{-1}$, about $17 \mathrm{~cm}^{-1}$ lower than for ${ }^{12} \mathrm{CH}_{4}$.

In the case of the $\mathrm{R}$ branch it was also possible to investigate the spectral region with the jet-CRD setup because the cw-diode laser of the setup covers this spectral region. Figure 6 shows the components of the R branch up to $J^{\prime \prime}=4$ recorded at room temperature (the cavity being used as a cell filled with a given amount of methane) as well as at very low rotational temperatures (below $10 \mathrm{~K}$ ) with the supersonic jet expansion. The FTIR spectrum recorded at $80 \mathrm{~K}$ is shown for comparison. We could not record the $\mathrm{R}(0)$ line with the jet-CRD setup with the $\mathrm{CH}_{4}$ : Ar gas mixture with $x\left({ }^{13} \mathrm{CH}_{4}\right)=0.02$ because the absorption was too strong and not enough light could be accumulated in the cavity to trigger the data acquisition process. Also the components with $\tilde{v}>7535.8 \mathrm{~cm}^{-1}$ for the $\mathrm{CH}_{4}$ : Ar 
Table 6: Line positions and assignment of the $P, Q$ and $R$ branches of the $v_{2}+2 v_{3}\left(F_{2}\right)$ combination band of ${ }^{13} \mathrm{CH}_{4}$. The symmetry of the rovibrational species is indicated for the $S_{4}^{*}$ group. The symmetry species of the levels are given as rovibrational species for ${ }^{13} \mathrm{CH}_{4}$ as for ${ }^{12} \mathrm{CH}_{4}$. For ${ }^{13} \mathrm{CH}_{4}$ all parities must be reversed $(+\leftrightarrow-)$ to obtain total parities including the ${ }^{13} \mathrm{C}$ nuclear spin function. The term values $E^{\prime}$ of the $v_{2}+2 v_{3}$ vibrational level are obtained from the line frequency with the given assignment and the term values of the vibrational ground state indicated in Table 5. The transitions indicated with a * are measured (and resolved) exclusively with the jet-CRD setup.

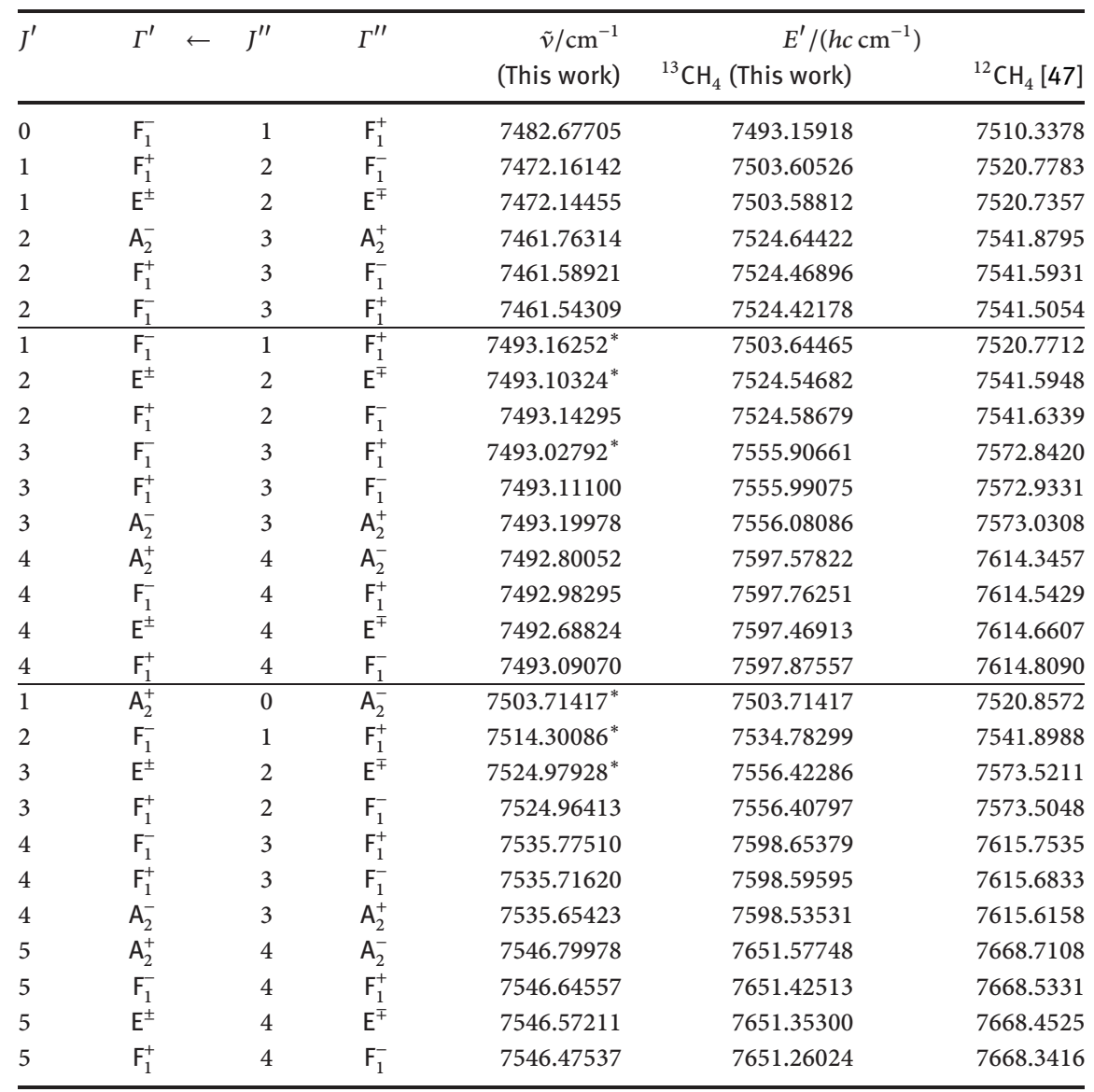

gas mixture with $x\left({ }^{13} \mathrm{CH}_{4}\right)=0.02$ and $\tilde{v}>7525.0 \mathrm{~cm}^{-1}$ for the $\mathrm{CH}_{4}$ : Ar gas mixture $x\left({ }^{13} \mathrm{CH}_{4}\right)<0.01$ were not measured because, in these cases, the absorption was too weak taking the intensity of the components with $\tilde{v}>7535.8 \mathrm{~cm}^{-1}$ into account. In all spectra the lines were fitted with a Gaussian profile and the resulting Full Width at Half Maximum (FWHM) varies from $0.0226 \mathrm{~cm}^{-1}$ at room tem- 


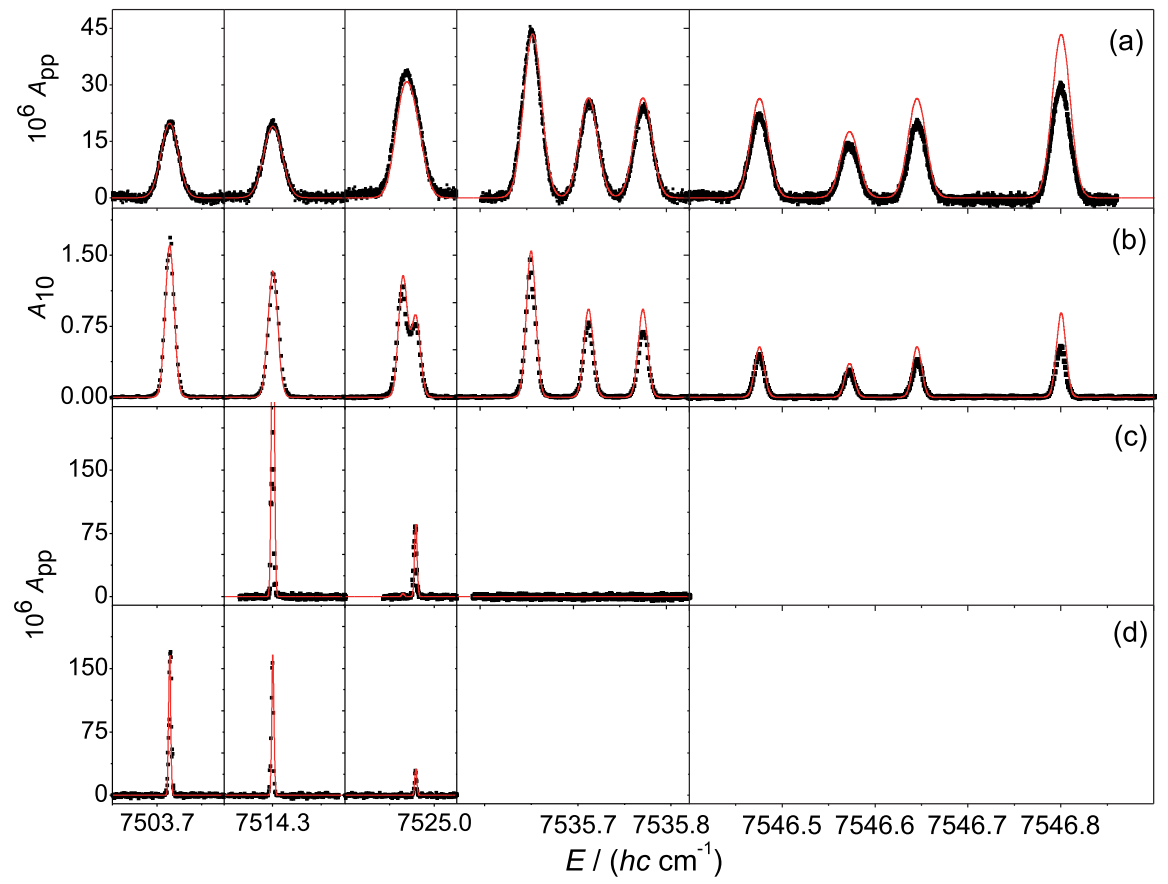

Figure 6: Spectra of the $\mathrm{R}$ branch of the $v_{2}+2 v_{3}$ combination band of methane ${ }^{13} \mathrm{CH}_{4}$ recorded for various rotational temperatures: (a) CRD spectrum at room temperature, (b) FTIR spectrum at $80 \mathrm{~K}$, and jet-CRD spectra of ${ }^{13} \mathrm{CH}_{4}$ : Ar gas mixtures with (c) $x\left({ }^{13} \mathrm{CH}_{4}\right)=0.02$ and (d) $x\left({ }^{13} \mathrm{CH}_{4}\right)<0.01$. The red lines are the simulated spectra at the estimated rotational temperature. CRD spectra are shown in Napierian absorbance per pass $A_{\mathrm{pp}}=\alpha \times L$ and FTIR spectrum in decadic absorbance $A_{10}=\log _{10}\left(I_{0} / I\right)$. The experimental conditions of the FTIR spectrum are as in Figure 1.

perature to $0.0024 \mathrm{~cm}^{-1}$ for the coldest CRD spectra recorded. Hence, the corresponding effective translational temperatures vary from $298 \mathrm{~K}$ down to $3 \mathrm{~K}$. The FTIR spectrum measured at $80 \mathrm{~K}$ is not quite Doppler limited with an approximate instrumental width $\delta \tilde{v}_{\text {instr. }} \simeq 0.005 \mathrm{~cm}^{-1}$ roughly estimated from the relation $\delta \tilde{v}_{\text {total }}=\sqrt{\delta \tilde{v}_{\text {Doppler }}^{2}+\delta \tilde{v}_{\text {instr. }}^{2}} \simeq 0.0123 \mathrm{~cm}^{-1}$ [33] and a Doppler width of about $\delta \tilde{v}_{\text {Doppler }} \simeq 0.012 \mathrm{~cm}^{-1}$ at $80 \mathrm{~K}$, assuming negligible pressure broadening under these conditions.

The first goal of these measurements at various temperatures is to confirm and reinforce the proposed assignment. Indeed each spectrum has been simulated using the values of the relative effective integrated absorption cross sections, as mentioned in Section 3.2. The rotational temperatures obtained are $T_{\text {rot }}=$ 
$298 \mathrm{~K}$ for the CRD spectrum (a) of Figure $6, T_{\text {rot }}=80 \mathrm{~K}$ for the FTIR spectrum (b), $T_{\text {rot }}<7 \mathrm{~K}$ and $T_{\text {rot }}=4 \mathrm{~K}$ for the jet-CRD spectra of ${ }^{13} \mathrm{CH}_{4}:$ Ar gas mixtures with $x\left({ }^{13} \mathrm{CH}_{4}\right)=0.02$ and $x\left({ }^{13} \mathrm{CH}_{4}\right)<0.01$, respectively. In the case of $T_{\text {rot }}<7 \mathrm{~K}$, we obtained an upper limit since only one line is not saturated and we could not compare the relative intensities. We based our estimation on the noise of the spectrum and the fact that the $\mathrm{R}\left(2, \mathrm{~F}_{1}^{-}\right)$component is not observable.

From $4 \mathrm{~K}$ to $298 \mathrm{~K}$, the simulated spectra shown in red in Figure 6 exhibit a good agreement with experiment. The two components $\mathrm{R}\left(2, \mathrm{E}^{ \pm}\right)$and $\mathrm{R}\left(2, \mathrm{~F}_{1}^{-}\right)$that coalesce at room temperature are just about resolved at $T_{\text {rot }}=80 \mathrm{~K}$ (FTIR spectrum) and can unambiguously be assigned with the two jet-CRD spectra at low rotational temperatures. In Figure 6 , the intensity of the $J^{\prime \prime}=4$ components seems to be not as nicely reproduced as that of the components with lower lower $J^{\prime \prime}$ values, although the relative intensities are in good agreement with those from experiments. This has been observed on two spectra obtained with different experimental conditions and different experimental setups. Therefore, any error arising from our setup can be excluded. Furthermore Figure 5 shows that similar relative intensities of the $J^{\prime \prime}=4$ components have been observed in the case of ${ }^{12} \mathrm{CH}_{4}$. This indicates that a local perturbation is quite improbable since the band is shifted by about $17 \mathrm{~cm}^{-1}$ for ${ }^{13} \mathrm{CH}_{4}$ compared to ${ }^{12} \mathrm{CH}_{4}$. One explanation would be that the simple model used for the relative intensity becomes less adequate for $J^{\prime \prime}=4$ already. The discrepancies do not prevent us, however, from giving a clear assignment in Table 6.

The CRD spectrum at room temperature allows for the estimation of the integrated band strength. Indeed, the integrated band strength $G_{\mathrm{ba}}$ is defined as the integrated absorption cross section over all rotational transitions of the vibrational band associated with the transition $\mathrm{b} \leftarrow \mathrm{a}$. In our case, a direct integration over all rovibrational transitions in the region of the $v_{2}+2 v_{3}$ combination band in the FTIR spectrum would certainly introduce some errors because of transitions in this region that do not belong to $v_{2}+2 v_{3}$ combination band but other bands of the icosad (see Figure 1). On the other hand, an isolated vibrational transition can, of course, not be strictly defined because of rovibrational interactions. Another possibility to get an estimation of $G_{\mathrm{b}}$ is to use the integrated absorption cross section $G\left(J_{\mathrm{f}}, \Gamma_{\mathrm{f}} \leftarrow J_{\mathrm{i}}, \Gamma_{\mathrm{i}}, T\right)$ of a rovibrational transition (f $\left.\leftarrow \mathrm{i}\right)$ or integrated rovibrational line strength belonging to this vibrational transition, which is actually proportional to $G_{\mathrm{ba}}[45,69-71]$ :

$$
G_{\mathrm{fi}}\left(J_{\mathrm{f}}, \Gamma_{\mathrm{f}} \leftarrow J_{\mathrm{i}}, \Gamma_{\mathrm{i}}, T\right)=\int_{\text {line of } \mathrm{f} \leftarrow \mathrm{i}} \sigma(T, \tilde{v}) \tilde{v}^{-1} \mathrm{~d} \tilde{v}=p_{\mathrm{i}}(T) R_{\mathrm{fi}} F d G_{\mathrm{ba}} .
$$

$p_{\mathrm{i}}(T)$ is the population of the lower state $\mathrm{i}$ of the transition at $T, R_{\mathrm{fi}}$ is the rotational line strength factor for the $J_{\mathrm{f}} \leftarrow J_{\mathrm{i}}$ transition, $F$ is a factor similar to the 


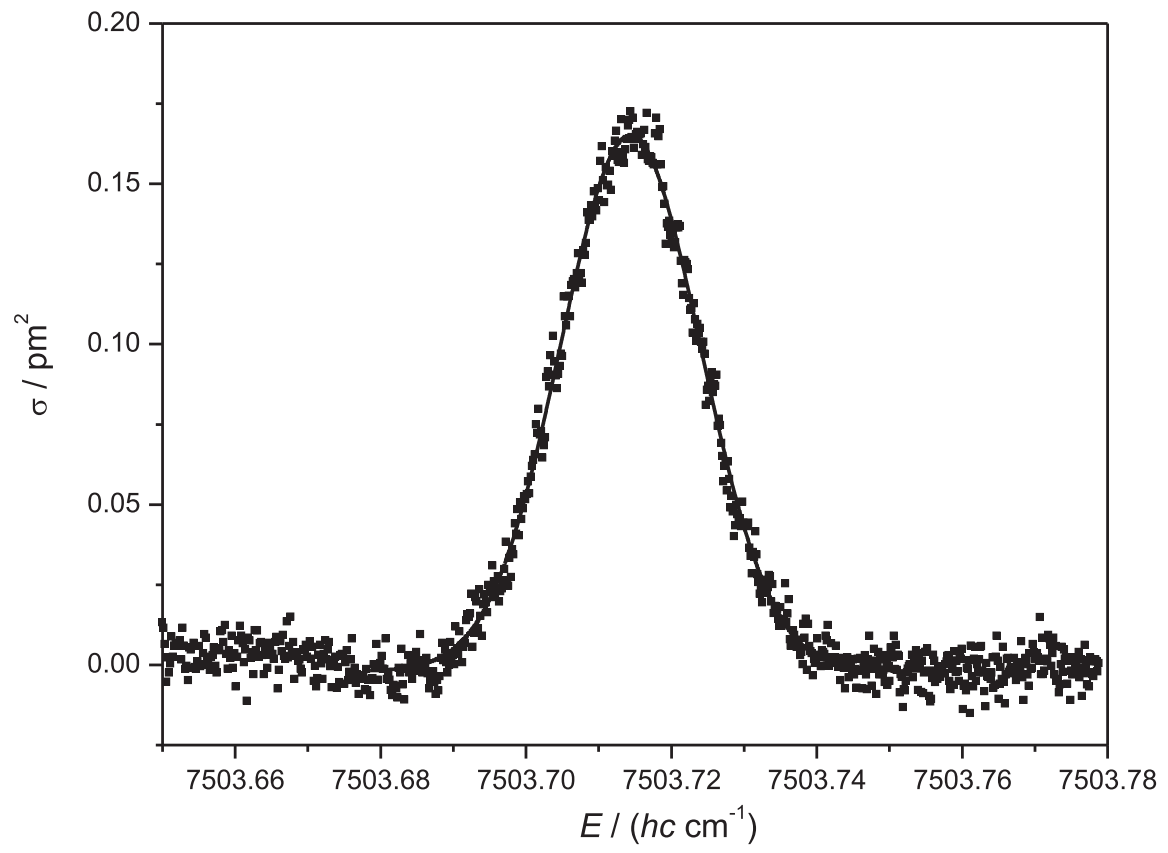

Figure 7: Effective absorption cross section $\sigma=C^{-1} \times L^{-1} \times \ln \left(I_{0} / I\right)$ (in $\mathrm{pm}^{2}$ ) of the $\mathrm{R}\left(0, \mathrm{~A}_{2}^{-}\right)$ transition of the $v_{2}+2 v_{3}$ combination band of ${ }^{13} \mathrm{CH}_{4}$ obtained from a CRD spectrum of $0.5903 \mathrm{mbar}$ of $\mathrm{C} \mathrm{CH}_{4}$ : Ar gas mixture with $x\left({ }^{13} \mathrm{CH}_{4}\right)=0.025$ at $298 \mathrm{~K}$ with $L=33 \mathrm{~cm}$. The full line corresponds to a fit with a Gaussian (Doppler) line shape with $\Delta \tilde{v}=0.0227 \mathrm{~cm}^{-1}$ (FWHM) with apparent Doppler temperature $304 \mathrm{~K}$.

Herman-Wallis factor for diatomic molecules and considers the transformation of the dipole moment operator due to rotation-vibration interactions; finally $d$ takes the dilution of the line strength into account by admixing dark state character by Coriolis interactions. At low $J$ values, Coriolis interactions are not very effective; $F$ and $d$ can then be considered as close to 1 and $R_{\mathrm{fi}}$ can be estimated with $\left(2 J_{\mathrm{f}}+1\right) /\left[3\left(2 J_{\mathrm{i}}+1\right)\right][45]$.

Figure 7 shows the absorption cross section of the $\mathrm{R}\left(0, \mathrm{~A}_{2}^{-}\right)$line of the $v_{2}+$ $2 v_{3}$ combination band of ${ }^{13} \mathrm{CH}_{4}$ obtained from the CRD spectrum at room temperature $(298 \mathrm{~K} \pm 1 \mathrm{~K})$, with $0.5903 \mathrm{mbar}$ of $\mathrm{a}^{13} \mathrm{CH}_{4}$ : Ar gas mixture with $x\left({ }^{13} \mathrm{CH}_{4}\right)=0.025$ in the cavity. The integrated absorption cross section has been estimated from three different measurements using various partial pressures ranging from $0.00576 \mathrm{mbar}$ to $0.0148 \mathrm{mbar}$ of methane ${ }^{13} \mathrm{CH}_{4}$ in a gas mixture with argon. From the $\mathrm{R}\left(0, \mathrm{~A}_{2}^{-}\right)$line, the integrated rovibrational line strength $G\left(1, \mathrm{~A}_{2}^{+} \leftarrow 0, \mathrm{~A}_{2}^{-}\right)$is estimated to be $(4.97 \pm 0.92) \times 10^{-7} \mathrm{pm}^{2}$. The uncertainty is indicated as $95 \%$ confidence interval for the three measurements, giving them 
equal weight. The resulting integrated band strength of the $v_{2}+2 v_{3}$ combination band is then $(5.32 \pm 0.99) \times 10^{-5} \mathrm{pm}^{2}$, about two times lower than that of ${ }^{12} \mathrm{CH}_{4}$ estimated at $(1.3 \pm 0.2) \times 10^{-4} \mathrm{pm}^{2}$ [45].

The integrated band strength $G_{\mathrm{ba}}$ is related to the vibrational transition moment $\mu_{\mathrm{b}}$ by the equation

$$
G_{\mathrm{ba}}=\left(\frac{1}{4 \pi \varepsilon_{0}}\right)\left(\frac{8 \pi^{3}}{3 h c}\right) 3\left|\mu_{\mathrm{ba}}\right|^{2} .
$$

Hence we estimate the vibrational transition moment to $\mu_{v_{2}+2 v_{3}}=(6.53 \pm 0.6) \times$ $10^{-4}$ Debye compared to $(1.0 \pm 0.1) \times 10^{-3}$ Debye for ${ }^{12} \mathrm{CH}_{4}$. This thus provides an estimate of both the band strength and the vibrational transition moment for the $v_{2}+2 v_{3}$ combination band of ${ }^{13} \mathrm{CH}_{4}$ which may be used as benchmarks for $a b$ initio calculations. Recent more extensive FTIR measurements in our laboratory give somewhat higher results than found here. We cannot entirely exclude some saturation effects or adsorption losses of ${ }^{13} \mathrm{CH}_{4}$ in our experiments.

\subsection{Analysis of the $Q$ branch and spectroscopic constants}

Figure 8 shows the FTIR spectrum of ${ }^{13} \mathrm{CH}_{4}$ in the region of the $\mathrm{Q}$ branch of the $v_{2}+2 v_{3}$ combination band recorded at $80 \mathrm{~K}$ with the Bruker ZP2001, together with that of ${ }^{12} \mathrm{CH}_{4}$ for comparison. At first sight, one can notice that the assignment would probably be more difficult than for the $\mathrm{P}$ and $\mathrm{R}$ branches because (i) the $\mathrm{Q}$ branch is more congested, all the components being concentrated in a range of about $1 \mathrm{~cm}^{-1}$ and (ii) the Q branch of $v_{2}+2 v_{3}$ combination band for ${ }^{13} \mathrm{CH}_{4}$ does not look like that for ${ }^{12} \mathrm{CH}_{4}$ so that our assignment can not simply rely on that obtained previously for ${ }^{12} \mathrm{CH}_{4}$. In that case, the use of spectra obtained at various temperatures plays a key role for the assignment as we demonstrated in our previous work [47].

Figure 9 shows the same FTIR spectrum obtained at $80 \mathrm{~K}$ (see the previous section for the determination of the rotational temperature) together with a CRD spectrum recorded at room temperature and two jet-CRD spectra of ${ }^{13} \mathrm{CH}_{4}$ : Ar gas mixtures with $x\left({ }^{13} \mathrm{CH}_{4}\right)=0.09$ and $x\left({ }^{13} \mathrm{CH}_{4}\right)<0.01$. From our investigation of the $\mathrm{R}$ branch, we expect these last two spectra to have a rotational temperature lower than $20 \mathrm{~K}$. The effective translational temperature, estimated from the Doppler width of $0.0038 \mathrm{~cm}^{-1}$ is $8 \mathrm{~K}$ and from $0.0032 \mathrm{~cm}^{-1}$, it is $6 \mathrm{~K}$.

The two cold spectra display the lowest $J$ components only and therefore strongly simplify the spectrum. Indeed the strongest component at $7493.16252 \mathrm{~cm}^{-1}$ is assigned to the $\mathrm{Q}\left(1, \mathrm{~F}_{1}^{+}\right)$transition and the second strongest at $7493.10324 \mathrm{~cm}^{-1}$ to the $\mathrm{Q}\left(2, \mathrm{E}^{ \pm}\right)$transition. In Figure $9(\mathrm{c})$, the $\mathrm{Q}\left(1, \mathrm{~F}_{1}^{+}\right)$transition is 

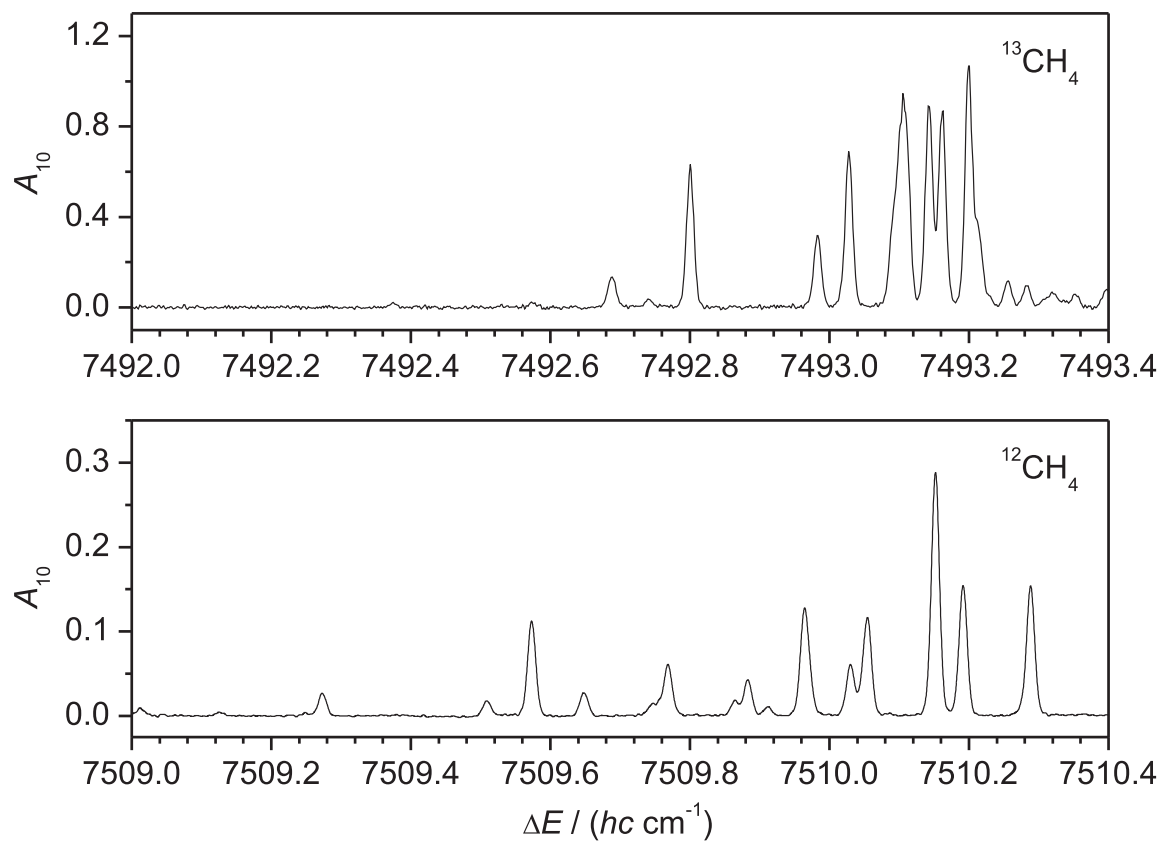

Figure 8: FTIR spectrum of the lowest components of the $\mathrm{Q}$ branch of the $v_{2}+2 v_{3}$ combination band of methane ${ }^{13} \mathrm{CH}_{4}$ (top) and ${ }^{12} \mathrm{CH}_{4}$ (bottom) for comparison. The experimental conditions are as in Figure 1. Both spectra were recorded at $80 \mathrm{~K}$ and are shown in decadic absorbance $A_{10}=\log _{10}\left(I_{0} / I\right)$ (see Reference [36]).

saturated on purpose under our experimental conditions to highlight a third component which appears weak: the line at $7493.14295 \mathrm{~cm}^{-1}$ is therefore assigned to the $\mathrm{Q}\left(2, \mathrm{~F}_{1}^{-}\right)$transition. With these three assignments as starting point, we can now analyse other lines of the spectra at higher temperature.

For example the intense line at $7493.19978 \mathrm{~cm}^{-1}$ that might have been assigned to one of the lowest $J$ values taking the spectra at $80 \mathrm{~K}$ and $298 \mathrm{~K}$ into account, actually belongs to a transition involving $J \geq 2$ considering the jet-CRD spectra at very low temperatures. Also the component around $7493.1 \mathrm{~cm}^{-1}$ is broad in the spectrum at $298 \mathrm{~K}$ and asymmetric in the spectrum at $80 \mathrm{~K}$. It results from a superposition of two transitions, one of which is still observable in the jet-CRD spectra at very low temperatures. We used our prediction of the relative integrated absorption cross sections as a function of the temperature to establish the assignment. The red lines in Figure 9 are the simulations of the spectra at the estimated rotational temperature according to the assignment given in Table 6 . Except for some slight discrepancies in the intensity of the $J=4$ components, as already discussed in the previous section in the case of the $\mathrm{R}$ branch, the line 


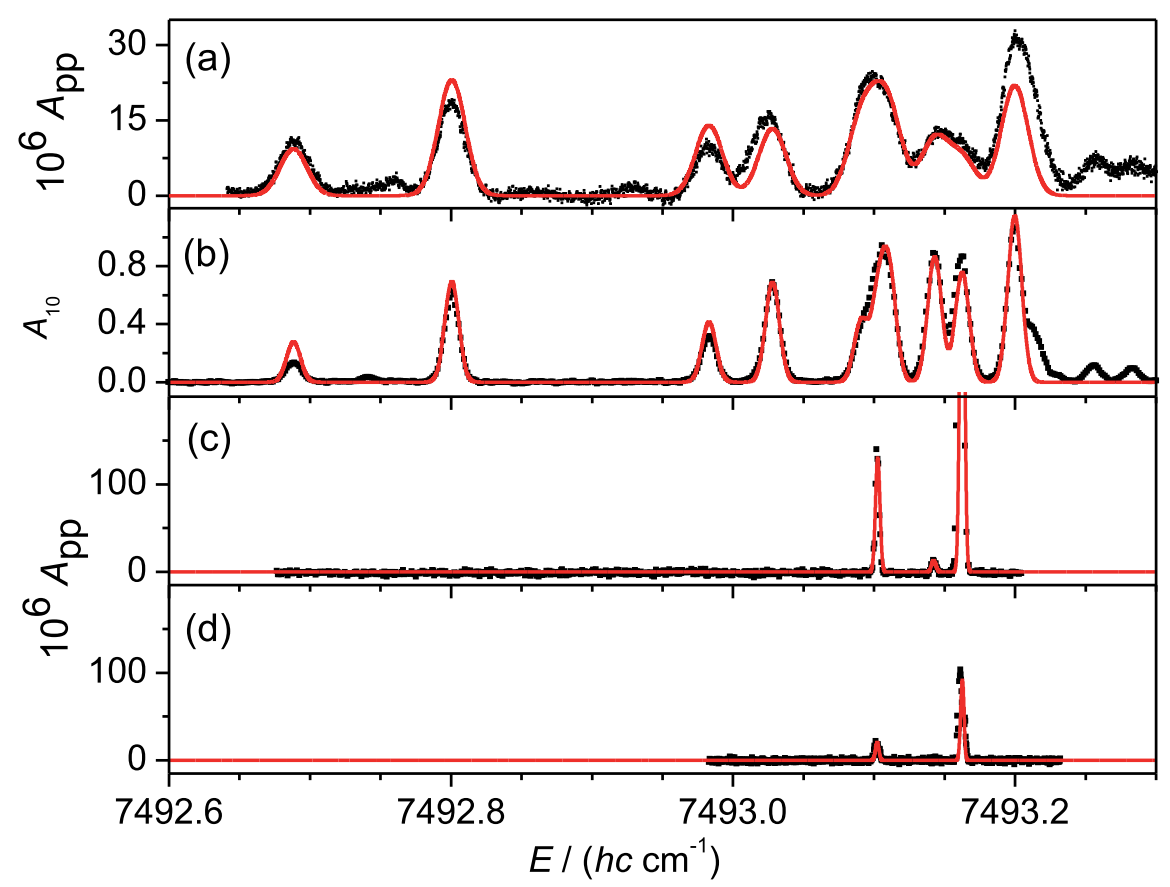

Figure 9: Spectra of the $\mathrm{Q}$ branch of the $v_{2}+2 v_{3}$ combination band of methane ${ }^{13} \mathrm{CH}_{4}$ recorded for various rotational temperatures: (a) CRD spectrum at room temperature, (b) FTIR spectrum at $80 \mathrm{~K}$, and jet-CRD spectra of ${ }^{13} \mathrm{CH}_{4}$ : Ar gas mixtures with (c) $x\left({ }^{13} \mathrm{CH}_{4}\right)=0.09$ and (d) $x\left({ }^{13} \mathrm{CH}_{4}\right)<0.01$. The red lines are the simulated spectra at the estimated rotational temperature. CRD spectra are shown in Napierian absorbance per pass $A_{\mathrm{pp}}=\alpha \times L$ and FTIR spectrum in decadic absorbance $A_{10}=\log _{10}\left(I_{0} / I\right)$.

strengths are well reproduced from $T_{\text {rot }}=4 \mathrm{~K}$ up to $298 \mathrm{~K}$. The intensity of the $\mathrm{Q}\left(3, \mathrm{~A}_{2}^{-}\right)$transition may seem poorly reproduced in the spectrum at $298 \mathrm{~K}$, since the simulation predicts a line strength significantly lower than found in the experiments, but in that case we believe that another component, not belonging to the $v_{2}+2 v_{3}$ combination band, overlaps. It is still observable as a "shoulder" in the spectrum at $80 \mathrm{~K}$ (Figure 9(b)) but not at lower temperatures (Figure 9(c) and (d)).

We have used the transitions of the $\mathrm{P}, \mathrm{Q}$ and $\mathrm{R}$ branches (up to $J=3$, for the $\mathrm{P}$ and $\mathrm{Q}$ branches and up to $J=4$ for the $\mathrm{R}$ branch) to estimate the effective spectroscopic constants of the $v_{2}+2 v_{3}$ band of ${ }^{13} \mathrm{CH}_{4}$ as discussed in [47]. Table 7 shows the calculated and experimental term values and Table 8 lists the values of the $a_{j}$, $b_{J}, c_{J}, d_{J}, e_{J}$, and $f_{J}$ parameters estimated from linear regressions following Equations (20)-(22). The rotational constant for the vibrational ground state of ${ }^{13} \mathrm{CH}_{4}$ is known [73] and $\tilde{B}_{0}=5.2412882 \mathrm{~cm}^{-1}$ is taken as a constant for the estimation 
Table 7: Experimental term values $E_{\exp }^{\prime}$ from Table 6 and those calculated from the fit $E_{\text {fit }}^{\prime}$. The difference $\Delta E^{\prime}=E_{\text {fit }}^{\prime}-E_{\text {exp }}^{\prime}$ is indicated for comparison.

\begin{tabular}{|c|c|c|c|c|}
\hline$J^{\prime}$ & $\Gamma^{\prime}$ & $E_{\text {exp }}^{\prime} /\left(h c \mathrm{~cm}^{-1}\right)$ & $E_{\text {fit }}^{\prime} /\left(h c \mathrm{~cm}^{-1}\right)$ & $\Delta E^{\prime} /\left(h c \mathrm{~cm}^{-1}\right)$ \\
\hline \multicolumn{5}{|c|}{$\mathrm{F}^{(+)}$subband } \\
\hline 1 & $\mathrm{E}^{ \pm}$ & 7503.58812 & 7503.58812 & 0.00000 \\
\hline 1 & $\mathrm{~F}_{1}^{+}$ & 7503.60526 & 7503.60526 & 0.00000 \\
\hline 2 & $\mathrm{~F}_{1}^{-}$ & 7524.42178 & 7524.43703 & 0.01525 \\
\hline 2 & $\mathrm{~F}_{1}^{+}$ & 7524.46896 & 7524.43465 & -0.03431 \\
\hline 2 & $\mathrm{~A}_{2}^{-}$ & 7524.64422 & 7524.44084 & 0.01906 \\
\hline \multicolumn{5}{|c|}{$\mathrm{F}^{(0)}$ subband } \\
\hline 2 & $\mathrm{E}^{ \pm}$ & 7524.54682 & 7524.54682 & 0.00000 \\
\hline 2 & $\mathrm{~F}_{1}^{+}$ & 7524.58679 & 7524.58679 & 0.00000 \\
\hline 3 & $\mathrm{~F}_{1}^{-}$ & 7555.90661 & 7555.90414 & -0.00247 \\
\hline 3 & $\mathrm{~F}_{1}^{+}$ & 7555.99075 & 7555.99520 & 0.00445 \\
\hline 3 & $\mathrm{~A}_{2}^{-}$ & 7556.08086 & 7556.07888 & -0.00198 \\
\hline \multicolumn{5}{|c|}{$\overline{\mathrm{F}^{(-)}}$subband } \\
\hline 3 & $\mathrm{~F}_{1}^{+}$ & 7556.40797 & 7556.40797 & 0.00000 \\
\hline 3 & $\mathrm{E}^{ \pm}$ & 7556.42286 & 7556.42286 & 0.00000 \\
\hline 4 & $\mathrm{~A}_{2}^{-}$ & 7598.53531 & 7598.53684 & -0.00153 \\
\hline 4 & $\mathrm{~F}_{1}^{+}$ & 7598.59595 & 7598.59939 & 0.00344 \\
\hline 4 & $\mathrm{~F}_{1}^{-}$ & 7598.65379 & 7598.65188 & -0.00191 \\
\hline 5 & $\mathrm{~F}_{1}^{+}$ & 7651.26024 & 7651.23264 & -0.02760 \\
\hline 5 & $\mathrm{E}^{ \pm}$ & 7651.35300 & 7651.39844 & 0.04544 \\
\hline 5 & $\mathrm{~F}_{1}^{-}$ & 7651.42513 & 7651.45267 & 0.02754 \\
\hline 5 & $\mathrm{~A}_{2}^{+}$ & 7651.57748 & 7651.53107 & -0.04641 \\
\hline
\end{tabular}

of the effective spectroscopic constants. We have found $\tilde{v}_{0}=7493.17(3) \mathrm{cm}^{-1}$, $\tilde{B}_{v_{2}+2 v_{3}}=5.256(2) \mathrm{cm}^{-1}, \tilde{D}_{v_{2}+2 v_{3}}=-5.1(14) \times 10^{-3} \mathrm{~cm}^{-1}$, and $\zeta_{v_{2}+2 v_{3}}=-0.003(1)$. The standard deviations indicated in parentheses in terms of the last significant digits take the uncertainties of the $a_{J}, b_{J}, c_{J}, d_{J}, e_{J}$, and $f_{J}$ parameters from the linear regressions into account. The effective band center estimated with this approach slightly differs from the "true" directly measured experimental value found at $7493.15918 \mathrm{~cm}^{-1}$ [36] and the value of $\tilde{D}_{v_{2}+2 v_{3}}$ has little meaning considering that only terms up to $J=3$ were taken into account. Here, we stress once more that the constants in Equations (17)-(19) have an interpretation as effective Hamiltonian constants only. This thus provides a first assignment and estimation of spectroscopic constants of the $v_{2}+2 v_{3}$ combination band of ${ }^{13} \mathrm{CH}_{4}$. A more complete analysis of the icosad is necessary to enlarge the assignment and provide more accurate spectroscopic constants, for which the present data can be a starting point (see also ref. [72]). 
Table 8: $a_{J}, b_{J}, c_{J}, d_{J}, e_{J}$ and $f_{J}$ parameters estimated from linear regressions (see Equations (20)-(22)) for the estimation of the effective spectroscopic constants $\tilde{v}_{0}, \tilde{B}_{2 v_{2}+2 v_{3}}$, $\tilde{D}_{2 v_{2}+2 v_{3}}$, and $\zeta_{2 v_{2}+2 v_{3}}$. The standard deviations are indicated in parentheses (in units of the last digits).

\begin{tabular}{lrrr}
\hline$J$ & 2 & 3 & 4 \\
\hline$a_{J} / \mathrm{cm}^{-1}$ & $-0.05544(-)$ & $-0.61(16)$ & \\
$b_{J} / \mathrm{cm}^{-1}$ & $7472.15467(-)$ & $7461.60(3)$ & \\
$c_{J} / \mathrm{cm}^{-1}$ & $0.0498(-)$ & $0.33(2)$ & $0.99(23)$ \\
$d_{J} / \mathrm{cm}^{-1}$ & $7524.97019(-)$ & $7535.733(3)$ & $7546.59(3)$ \\
$e_{J} / \mathrm{cm}^{-1}$ & $-0.1305(-)$ & $-0.46(2)$ & \\
$f_{J} / \mathrm{cm}^{-1}$ & $7493.12707(-)$ & $7493.088(3)$ & \\
\hline
\end{tabular}

\subsection{Nuclear spin symmetry conservation}

This aspect is an important part of the present work on ${ }^{13} \mathrm{CH}_{4}$ as well. The conservation of nuclear spin symmetry in ${ }^{12} \mathrm{CH}_{4}$ has already been investigated using a supersonic jet expansion [47-49] as well as an enclosive flow cooling in our group [37, 50] and in further work with diode laser spectroscopy [74] (see also the review in [62]). It has been found quite generally that the nuclear spin symmetry is conserved in the supersonic jet expansion (for ${ }^{13} \mathrm{CH}_{4}$ there existed so far no such investigations concerning nuclear spin symmetry conservation). In our recent investigation on ${ }^{12} \mathrm{CH}_{4}$, we have shown that temperatures well below $40 \mathrm{~K}$ must be achieved in order to obtain firm conclusions upon nuclear spin symmetry conservation for methane from measurements of line intensities [47]. Therefore, only the jet-CRD spectra below $10 \mathrm{~K}$ will be considered here.

Figure 10(b) shows the coldest jet-CRD spectra obtained at $4 \mathrm{~K}$ in the region of the $\mathrm{Q}$ branch (top) and the $\mathrm{R}$ branch (bottom) of the $v_{2}+2 v_{3}$ combination band. The simulation of the branches in the case of nuclear spin symmetry relaxation (a) and nuclear spin symmetry conservation (c) are shown for comparison, the simulated spectra being "scaled" to the intensity of the strongest line of the experimental spectrum for the corresponding bands. In both cases, our results clearly show that nuclear spin symmetry is conserved in ${ }^{13} \mathrm{CH}_{4}$, since the relative intensity of the lines with $J \geq 0$ or 1 can not be reproduced in the case of nuclear spin symmetry relaxation. This result is in agreement with measurements with previous investigations on ${ }^{12} \mathrm{CH}_{4}$, and more generally the theory of nuclear spin symmetry conservation in a supersonic expansion, as mentioned above. 

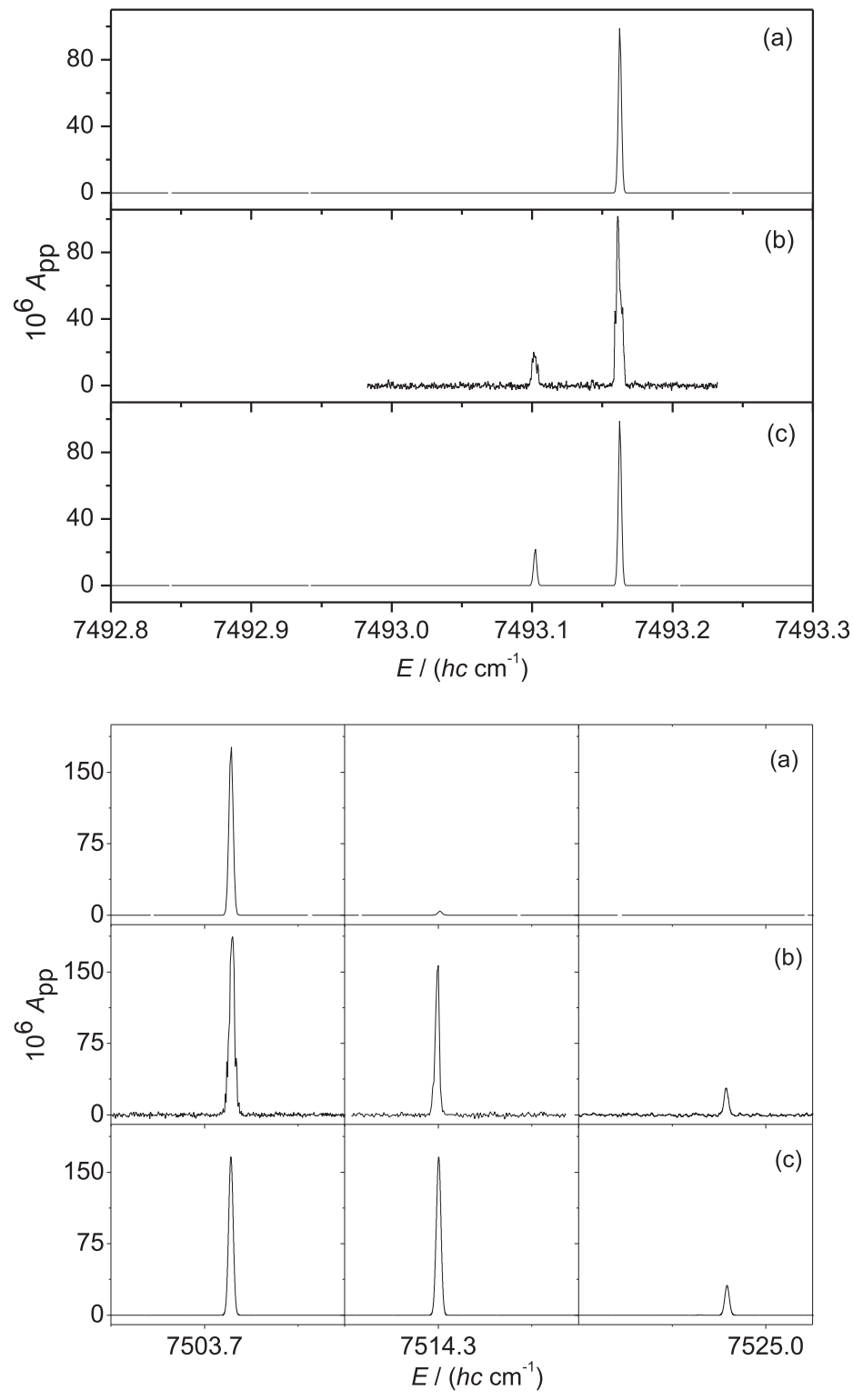

Figure 10: Lowest components of the $\mathrm{Q}$ (top) and $\mathrm{R}$ (bottom) branches of the $v_{2}+2 v_{3}$ combination band of ${ }^{13} \mathrm{CH}_{4}$ at $4 \mathrm{~K}$ : (a) Simulation in the case of nuclear spin symmetry relaxation, (b) jet-CRD spectrum of ${ }^{13} \mathrm{CH}_{4}$ : Ar gas mixture with $x\left({ }^{13} \mathrm{CH}_{4}\right)<0.01$, and (c) simulation in the case of nuclear spin symmetry conservation. The intensity of the simulations has been arbitrarily calibrated to the most intense line of the corresponding experimental spectrum for comparison. 


\section{Discussion and conclusions}

The question of nuclear spin symmetry relaxation or conservation in supersonic expansions had previously not been addressed for ${ }^{13} \mathrm{CH}_{4}$. The recent developments in our work on the main isotope of methane ${ }^{12} \mathrm{CH}_{4}$ [47] and on water $\mathrm{H}_{2}^{16} \mathrm{O}$ [66] using the improved jet-CRD setup have allowed us here to carry out such a study for ${ }^{13} \mathrm{CH}_{4}$ very efficiently reaching very low temperatures of about $4 \mathrm{~K}$. Our studies have proven our capacity of performing temperature-dependent investigations and obtaining semi-quantitative measurements of populations of rovibrational levels in a supersonic jet expansion with this setup. Here, we have used it also to investigate the $v_{2}+2 v_{3}$ subband in the overtone icosad of ${ }^{13} \mathrm{CH}_{4}$, with the same approach. This has allowed us to achieve two goals.

The first goal is the contribution to the understanding of the high resolution rovibrational spectroscopy of methane, and more specifically of the ${ }^{13} \mathrm{CH}_{4}$ isotopomer, for which significantly less is known compared with the ${ }^{12} \mathrm{CH}_{4}$ main isotopomer. We propose a first assignment of the lowest $J$ components for the $v_{2}+2 v_{3}$ subband and an estimate of the spectroscopic constants as well as band strengths and transition dipole moments, which can be considered as a starting point for a possible more complete analysis of the region, including treatment of couplings with other states.

Regarding our second goal, we have investigated the questions of nuclear spin symmetry conservation or relaxation in ${ }^{13} \mathrm{CH}_{4}$ at very low temperatures (down to $4 \mathrm{~K}$ ). Our results demonstrate that nuclear spin symmetry is conserved, in agreement with investigations performed on the main isotopomer and with the theory of supersonic jet expansions in relation to nuclear spin symmetry relaxation. While expected, this result nevertheless needed experimental proof, as the difference in energy level patterns of ${ }^{12} \mathrm{CH}_{4}$ and ${ }^{13} \mathrm{CH}_{4}$ could lead to significant changes and surprises due to the different spin and parity of ${ }^{13} \mathrm{C}$ nucleus might arise as well [58].

Nuclear spin symmetry conservation and relaxation might also be introduced in the most detailed treatments of the roles of the corresponding time scales for symmetry breaking in dissociation and recombination kinetics in general [58] and for methane and its isotopomers in particular, as it continues to be a prototypical system for reaction dynamics until the most recent times [76], including gas phase activation and catalysis [77].

Acknowledgement: Substantial help from and discussions with Sieghard Albert and Irina Bolotova are gratefully acknowledged. We also enjoyed useful exchange on the methane spectra with Hans Martin Niederer, Sigurd Bauerecker, Vincent 
Boudon, Tucker Carrington, Michael Rey, Elena Bekhthereva, Olga Gromova and Oleg Ulenikov. Andreas Schneider and André Laso provided important technical help. Our work is supported financially by ETH Zürich, the Schweizerischer Nationalfonds für Förderung der wissenschaftlichen Forschung and the European Research Council by an Advanced Grant. Our work is also to be seen in the context of the COST project MOLIM. The research leading to these results has also received funding from the European Research Council under the European Union's Seventh Framework Program (FP7, 2007-2013) ERC Grant No 290925.

This paper is dedicated to Jürgen Troe on the occasion of his $75^{\text {th }}$ birthday, remembering numerous discussions on methane kinetics and dynamics during more than four decades.

\section{References}

1. J. H. van't Hoff, Vorlesungen über theoretische und physikalische Chemie, Band 2, Vieweg, Braunschweig (1899).

2. L. Pauling, The Nature of the Chemical Bond, second edn., Cornell University Press, New York (1940).

3. M. Quack and J. Troe, Ber. Bunsen. Phys. Chem. 78 (1974) 240.

4. M. Quack and J. Troe, Ber. Bunsen. Phys. Chem. 79 (1975) 170.

5. M. Quack and J. Troe, Unimolecular reactions and energy transfer of highly excited molecules, in Gas Kinetics and Energy Transfer, Vol. 2, P. G. Ashmore, R. J. Donovan (Eds.), The Chemical Society London, ISBN 0851867669 (1977), pp. 175-238.

6. S. Peyerimhoff, M. Lewerenz, and M. Quack, Chem. Phys. Lett. 109 (1984) 563.

7. M. Lewerenz and M. Quack, J. Chem. Phys. 88 (1988) 5408.

8. M. Quack, Ann. Rev. Phys. Chem. 41 (1990) 839.

9. R. Marquardt and M. Quack, J. Chem. Phys. 109 (1998) 10628.

10. R. Marquardt and M. Quack, J. Phys. Chem. A 108 (2004) 3166.

11. R. Marquardt and M. Quack, chapter Global Analytical Potential Energy Surfaces for High-resolution Molecular Spectroscopy and Reaction Dynamics, in: Handbook of HighResolution Spectroscopy, Volume 1, M. Quack, F. Merkt, Eds., Wiley, Chichester (2011), pp. 511-549.

12. F. Merkt and M. Quack, chapter Molecular Quantum Mechanics and Molecular Spectra, Molecular Symmetry, and Interaction of Matter with Radiation, in: Handbook of HighResolution Spectroscopy, Volume 1, M. Quack, F. Merkt (Eds.), Wiley, Chichester (2011), pp. $1-55$.

13. S. Albert, K. Keppler Albert, H. Hollenstein, C. Manca Tanner, and M. Quack, chapter Fundamentals of Rotation-Vibration Spectra, in: Handbook of High-Resolution Spectroscopy, Volume 1, M. Quack, F. Merkt (Eds.), Wiley, Chichester (2011), pp. 117-173 .

14. M. Quack, European Review 22 (2014) S50.

15. M. Quack, J. Phys. Chem. 83 (1979) 150.

16. C. J. Cobos and J. Troe, Chem. Phys. Lett. 113 (1985) 419. 
17. M. Quack and J. Troe, Statistical Adiabatic Channel Models, in: Encyclopedia of Computational Chemistry, Vol. 4, P. von Ragué Schleyer, N. Allinger, T. Clark, J. Gasteiger, P. A. Kollman, H. F. Schaefer III, P. R. Schreiner (Eds.), John Wiley and Sons, Chichester, UK (1998), p. 2708-2726.

18. X.-G. Wang and T. J. Carrington Jr., J. Chem. Phys. 119 (2003) 101, and supplementary material (EPAPS) available at http://ftp.aip.org/epaps/j_chem_phys/E-JCPSA6-118-024323/.

19. X.-G. Wang and T. J. Carrington Jr., J. Chem. Phys. 141 (2014) 154106.

20. T. Carrington Jr., chapter Using Iterative Methods to Compute Vibrational Spectra, in: Handbook of High-Resolution Spectroscopy, Volume 1, M. Quack, F. Merkt (Eds.), Wiley, Chichester (2011), pp. 573-585.

21. A. G. Császár, C. Fábri, T. Szidarovszky, E. Mátyus, T. Furtenbacher, and G. Czakó, Phys. Chem. Chem. Phys. 14 (2012) 1085.

22. R. Marquardt and M. Quack, J. Chem. Phys. 95 (1991) 4854.

23. R. Marquardt, M. Quack and I. Thanopulos, J. Phys. Chem. A 104 (2000) 6129.

24. R. Marquardt and M. Quack, chapter A.3.13, in: Encyclopedia of Chemical Physics and Physical Chemistry, Vol. 1 (Fundamentals), J. H. Moore, N. D. Spencer (Eds.), IOP Publ., Bristol (2001), pp. 897-936.

25. M. Quack, Chimia 55 (2001) 753; Il Nuovo Cimento 63B (1981) 358.

26. M. Quack, Angew. Chem. Int. Edit. 28 (1989) 571.

27. M. Pepper, I. Shavitt, P. Ragué Schleyer, M. Glukhovtsev, R. Janoschek, and M. Quack, J. Comp. Chem. 16 (1995) 207.

28. A. Bakasov, T. K. Ha, and M. Quack, J. Chem. Phys. 109 (1998) 7263.

29. M. A. K. Khalil, Annu. Rev. Energ. Env. 24 (1999) 645.

30. A. Coustenis, E. Gendron, O. Lai, J. P. Veran, J. Woillez, M. Combes, L. Vapillon, T. Fusco, and L. Mugnier, Icarus 154 (2001) 501.

31. A. Negrao, A. Coustenis, E. Lellouch, J. P. Maillard, P. Rannou, B. Schmitt, C. P. McKay, and V. Boudon, Planet. Space Sci. 554 (2006) 1225.

32. S. Albert, S. Bauerecker, V. Boudon, L. R. Brown, J. P. Champion, M. Loete, A. Nikitin, and M. Quack, Chem. Phys. 356 (2009) 131.

33. S. Albert, K. Keppler Albert, and M. Quack, chapter High-resolution Fourier Transform Infrared Spectroscopy, in: Handbook of High-Resolution Spectroscopy, Volume 2, M. Quack, F. Merkt (Eds.), Wiley, Chichester (2011), pp. 965-1019.

34. A. V. Nikitin, V. Boudon, L. R. Wenger, C. and L. Brown, S. Bauerecker, S. Albert, and M. Quack, Phys. Chem. Chem. Phys. 15 (2013) 10071; see also paper L30, p. 288 in Proceedings of the 22nd Colloquium on High-Resolution Molecular Spectroscopy, Dijon (2011).

35. H. M. Niederer, S. Albert, S. Bauerecker, V. Boudon, J. P. Champion, and M. Quack, Chimia $62(2008) 273$.

36. O. N. Ulenikov, E. S. Bekhtereva, S. Albert, S. Bauerecker, H. M. Niederer, and M. Quack, J. Chem. Phys. 141 (2014) 234302; see also paper 010, p. 359-360 in Proceedings of the 22nd Colloquium on High-Resolution Molecular Spectroscopy, Dijon (2011).

37. H. M. Niederer, The Infrared Spectrum of Methane, Dissertation no 19829, ETH Zurich, Zürich, Switzerland (2012), ISBN 978-3-8439-0459-9.

38. H.-M. Niederer, X. G. Wang, T. Carrington Jr, S. Albert, S. Bauerecker, V. Boudon and M. Quack, J. Mol. Spectrosc. 291 (2013) 33.

39. V. Boudon, J. P. Champion, T. Gabard, M. Loëte, M. Rotger, and C. Wenger, chapter Spherical Top Theory and Molecular Spectra, in: Handbook of High-Resolution Spectroscopy, Volume 2, M. Quack, F. Merkt (Eds.), Wiley, Chichester (2011), pp.1437-1460. 
40. G. Herzberg, Molecular Spectra and Molecular Structure, Vol. II, Infrared and Raman Spectra of Polyatomic Molecules, 1st edn., van Nostrand Reinhold, New York (1945) and Vol. III, Electronic Spectra of Polyatomic Molecules, 1st edn., van Nostrand Reinhold, New York (1966).

41. D. N. Kozlov, A. M. Prokhorov, and V. V. Smirnov, J. Mol. Spectrosc. 77 (1979) 21.

42. V. Boudon, M. Rey, and M. Loëte, J. Quant. Spectrosc. Ra. 98 (2006) 394.

43. A. G. Robiette, J. Mol. Spectrosc. 86 (1981) 143.

44. D. L. Gray, A. G. Robiette, and A. S. Pine, J. Mol. Spectrosc. 77 (1979) 440.

45. M. Hippler and M. Quack, J. Chem. Phys. 116 (2002) 6045.

46. M. Hippler and M. Quack, Chem. Phys. Lett. 314 (1999) 273.

47. C. Manca Tanner and M. Quack, Mol. Phys. 110 (2012) 2111.

48. A. Amrein, M. Quack, and U. Schmitt, J. Phys. Chem. 92 (1988) 5455.

49. H. R. Dübal, M. Quack, and U. Schmitt, Chimia 38 (1984) 438.

50. H. M. Niederer, S. Albert, S. Bauerecker, V. Boudon, G. Seyfang, and M. Quack, Faraday Discuss. 150 (2011) 128.

51. R. F. J. Curl, J. V. V. Kasper, and K. Pitzer, J. Chem. Phys. 46 (1967) 3220; F. H. Frayer and G. E. Ewing, J. Chem. Phys. 48 (1967) 781; L. H. Jones, S. A. Ekberg, and B. I. Swanson, J. Chem. Phys. 85 (1986) 3203.

52. P. L. Chapovsky and L. J. F. Hermans, Ann. Rev. Phys. Chem. 50 (1999) 315.

53. C. Manca Tanner, Z. Bjelobrk and M. Quack, paper D15, p. 82 in Proceedings of the 23rd Colloquium on High-Resolution Molecular Spectroscopy, Budapest (2013).

54. C. Manca Tanner, Z. Bjelobrk and M. Quack, Chimia 68 (Supplementa to Issue 7/8) (2014) $\mathrm{PC}-134$.

55. Y. He, M. Hippler and M. Quack, Chem. Phys. Lett. 289 (1998) 527.

56. H. C. Longuet-Higgins, Mol. Phys. 6 (1963) 445.

57. M. Quack, Mol. Phys. 34 (1977) 477.

58. M. Quack, chapter Fundamental Symmetries and Symmetry Violations from Highresolution Spectroscopy, in: Handbook of High-Resolution Spectroscopy, Volume 1, M. Quack, F. Merkt (Eds.), Wiley, Chichester (2011), pp. 659-722.

59. J. T. Hougen, J. Chem. Phys. 37 (1962) 1433.

60. J. T. Hougen, J. Chem. Phys. 55 (1971) 1122.

61. M. Herman, R. Georges, M. Hepp, and D. Hurtmans, Int. Rev. Phys. Chem. 19 (2000) 277.

62. M. Snels, V. Horká-Zelenková, H. Hollenstein, and M. Quack, chapter High-resolution FTIR and Diode Laser Spectroscopy of Supersonic Jets, in: Handbook of High-Resolution Spectroscopy, Volume 2, M. Quack, F. Merkt (Eds.), Wiley, Chichester (2011), pp. 1021-1067.

63. I. Ozier and P.-N. Yi, J. Chem. Phys. 47 (1967) 5458.

64. I. Ozier, P.-N. Yi, A. Khosla, and N. Ramsey, Phys. Rev. Lett. 24 (1970) 642.

65. M. Hippler, M. Oeltjen, and M. Quack, J. Phys. Chem. A 111 (2007) 12659, and to be published.

66. C. Manca Tanner, M. Quack, and D. Schmidiger, Faraday Discuss. 150 (2011) 118; J. Phys. Chem. A 117 (2013) 10105.

67. J. Moret-Bailly, J. Mol. Spectrosc. 15 (1965) 344.

68. J. Moret-Bailly, L. Gautier, and J. Montagutelli, J. Mol. Spectrosc. 15 (1965) 355.

69. M. Dang-Nhu, A. S. Pine, and A. G. Robiette, J. Mol. Spectrosc. 77 (1979) 57.

70. K. Fox, Phys. Rev. A 8 (1973) 658.

71. K. Fox and W. B. Person, J. Chem. Phys. 64 (1976) 5218. 
72. V. Boudon, M. Loëte, M. Hippler, M. Quack, M. Rey, paper L36, p. 375-376 in Proceedings of the 18th Colloquium on High-Resolution Spectroscopy, Dijon (2003).

73. J. P. Champion, J. C. Hilico and L. R. Brown, J. Mol. Spectrosc. 133 (1989) 244.

74. M. Hepp, G. Winnewisser, and K. Yamada, J. Mol. Spectrosc. 164 (1994) 311.

75. C. Wenger, V. Boudon, M. Rotger, M. Sanzharov, and J.-P. Champion J. Mol. Spectrosc. 251 (2008) 102.

76. J. Troe, Mol. Phys. 112 (2014) 2374.

77. H. Schwarz, Isr. J. Chem. 54 (2014) 1431. 